\title{
Highly diastereoselective boron and titanium mediated aldol reactions of a mannitol derived 2,3-butanediacetal ethyl ketone.
}

Matthew O'Brien, ${ }^{* a}$ Arne Weber, ${ }^{b}$ Gemma Hardy $^{\mathrm{b}}$ and Steven V. Ley ${ }^{\mathrm{b}}$.

${ }^{a}$ Lennard-Jones Laboratories, School of Chemical and Physical Sciences, Keele University, Borough of Newcastle-under-Lyme, Staffordshire, UK, ST5 5BG.

${ }^{b}$ Chemistry Department, University of Cambridge, Lensfield Road, Cambridge, UK, CB2 $1 E W$.

\begin{abstract}
A mannitol derived 2,3-butanediacetal ethyl ketone displays high levels of diastereoselectivity in boron and titanium mediated aldol reactions with a range of aliphatic and aromatic aldehydes to afford syn aldol products in high yield. The stereochemical outcome of the reaction was determined using $J$-value analysis, NMR analysis of $O$ acetylmandelate derivatives and X-ray crystallography.
\end{abstract}

\section{Introduction}

Since its discovery by Borodin, ${ }^{1}$ and independently by Wurtz, ${ }^{2}$ the aldol addition reaction has become one of the most important transformations in organic synthesis. ${ }^{3}$ Its ability to furnish $\beta$-hydroxy carbonyl compounds with excellent levels of stereocontrol has led to its widespread employment in the total synthesis of natural products, particularly secondary metabolites derived from the polyketide biosynthetic pathway. ${ }^{4}$

As part of our research exploring the synthetic utility of 2,3-butane-diacetal derivatives of polyol precursors, ${ }^{5}$ we have previously shown that a simple synthetic sequence can be used to convert D-mannitol (1), via the selectively functionalised intermediate $\mathbf{2}$ to either the equatorial (3) or axial (5) diastereoisomer of (5R,6R)-5,6-dimethoxy-5,6-dimethyl-1,4dioxane-2-carbaldehyde at will (Scheme 1). ${ }^{6}$

In many of the reactions in which they act as electrophiles, these aldehydes display excellent stereodirecting abilities, giving rise to a highly versatile stereochemical manifold. ${ }^{7}$ Building on this, we wished to explore the use of the 2,3-butane diacetal motif as the stereodirecting 
group in a nucleophilic reaction partner and chose the aldol reaction as our initial subject of investigation. To this end, the equatorial ethyl ketone $\mathbf{4}$ was synthesised in high yield from $\mathbf{3}$ using a simple Grignard-addition/Swern-oxidation sequence. In this manuscript, we report that boron and titanium mediated aldol reactions of $\mathbf{4}$ proceed with excellent levels of diastereoselectivity, furnishing syn,syn products with a range of aldehydes.

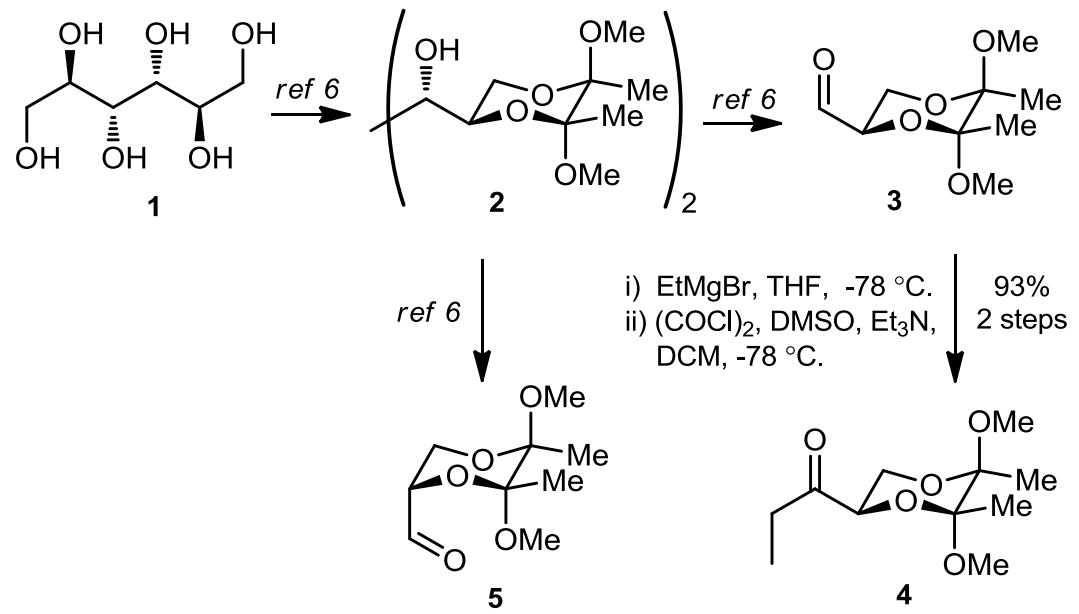

Scheme 1 Reagents and conditions: i. EtMgBr, THF, $-78{ }^{\circ} \mathrm{C}$. ii. $(\mathrm{COCl})_{2}, \mathrm{Me}_{2} \mathrm{SO}, \mathrm{Et}_{3} \mathrm{~N}$, $\mathrm{DCM},-78^{\circ} \mathrm{C}$.

We began our investigation with benzaldehyde, $\mathbf{6 a}$, as the electrophilic reaction partner. Initially exploring the generation of dialkyl boron enolates, a brief survey of reagents and conditions identified dicyclohexylboron chloride and triethylamine in diethyl ether at $-60{ }^{\circ} \mathrm{C}$ as suitable, giving rise to high levels of stereocontrol whilst allowing the reaction to proceed at an acceptable rate.

Under these conditions, using recently prepared dicyclohexyl boron chloride, ${ }^{8}$ the coupling of 4 with benzaldehyde afforded a $98 \%$ yield of the product $7 \mathbf{a}$ in a diastereomeric ratio of $96: 4$ as determined from ${ }^{1} \mathrm{H}$ NMR spectroscopy (Scheme 2, Table 1). Using the same conditions, 4 was then coupled with a series of aromatic and aliphatic aldehydes to afford the corresponding aldol products $\mathbf{7 a - j}$ in excellent yields and with very high levels of stereocontrol (Scheme 2, Table 1). An oxidative workup with alkaline hydrogen peroxide was used to convert the cyclohexyl groups on boron to cyclohexanol prior to chromatographic purification. 


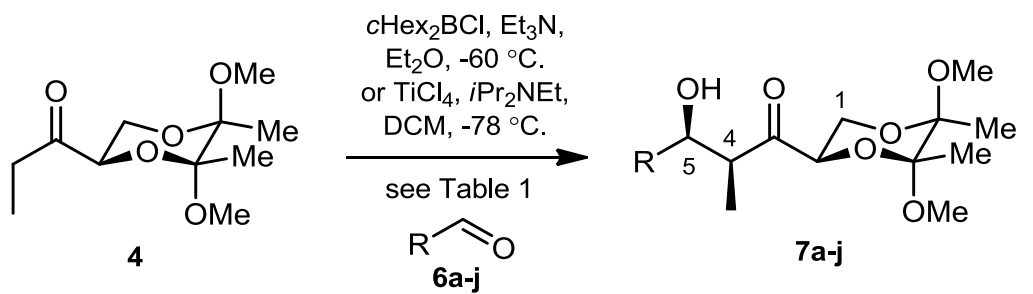

Scheme 2 Reagents and conditions: 4, $c \mathrm{Hex}_{2} \mathrm{BCl}, \mathrm{Et}_{3} \mathrm{~N}, \mathrm{Et}_{2} \mathrm{O},-60{ }^{\circ} \mathrm{C}$ then $\mathbf{6 a - j}, \mathrm{Et}_{2} \mathrm{O}-60{ }^{\circ} \mathrm{C}$ OR 4, $\mathrm{TiCl}_{4}, \mathrm{iPr}_{2} \mathrm{NEt}, \mathrm{CH}_{2} \mathrm{Cl}_{2},-78{ }^{\circ} \mathrm{C}$, then 6a-c.

Table 1 Results of the aldol coupling of 4 .

\begin{tabular}{|c|c|c|c|c|c|c|c|}
\hline entry & 6 & Aldehyde & Product & Method $^{\mathrm{a}}$ & $\begin{array}{c}{ }^{3} J_{\mathrm{HH}} 4,5 \\
(\mathrm{~Hz})\end{array}$ & $\begin{array}{c}\text { Yield }^{\mathrm{b}} \\
(\%)\end{array}$ & d.r. \\
\hline 1 & \multirow{2}{*}{$6 \mathbf{a}$} & & \multirow{2}{*}{$7 \mathbf{a}$} & B & 4.6 & 98 & $96: 4$ \\
\hline 2 & & & & $\mathrm{Ti}$ & $"$ & 95 & $95: 5$ \\
\hline 3 & \multirow{2}{*}{$6 b$} & & \multirow{2}{*}{$7 b$} & B & 4.4 & 94 & $95: 5$ \\
\hline 4 & & & & $\mathrm{Ti}$ & $"$ & 92 & $94: 6$ \\
\hline 5 & \multirow{2}{*}{$6 c$} & & \multirow{2}{*}{$7 c$} & B & 2.9 & 98 & $94: 6$ \\
\hline 6 & & & & $\mathrm{Ti}$ & $"$ & 91 & $94: 6$ \\
\hline 7 & 6d & & 7d & B & 3.6 & 92 & $95: 5$ \\
\hline 8 & $6 e$ & & $7 e$ & B & 3.1 & 97 & $97: 3$ \\
\hline 9 & $6 f$ & & $7 f$ & B & 3.2 & 96 & $94: 6$ \\
\hline 10 & $6 \mathrm{~g}$ & & $7 g$ & B & 2.8 & 93 & $94: 6$ \\
\hline 11 & $6 h$ & & $7 \mathrm{~h}$ & B & 3.5 & 94 & $95: 5$ \\
\hline 12 & $6 \mathbf{i}$ & & $7 \mathbf{i}$ & B & 4.3 & 95 & $94: 6$ \\
\hline 13 & $6 \mathbf{j}$ & & $7 \mathbf{j}$ & B & 6.5 & 97 & $95: 5$ \\
\hline
\end{tabular}

${ }^{\mathrm{a}} \mathrm{B}=$ Boron, $\mathrm{Ti}=$ Titanium. $^{\mathrm{b}}$ isolated yield after purification by column chromatography on silica gel.

The relatively low $\alpha-\beta{ }^{1} \mathrm{H}-{ }^{1} \mathrm{H}$ coupling constants (Table $1,4^{\text {th }}$ column) are consistent with the newly created stereocentres having a relative syn relationship, according to the Stiles-House model. ${ }^{9}$ Although dicyclohexylboron chloride often leads to the formation of $E$-boron enolates, the enolate geometry is highly dependent on the substitution pattern at the $\alpha$ 
position and related $\alpha^{\prime}$-alkoxy ketones have been shown to afford exclusive formation of $Z$ boron enolates using this reagent. ${ }^{10}$

The absolute configuration of the $\beta$ hydroxyl stereocentre in the aldol products was determined by comparison of the ${ }^{1} \mathrm{H}$ NMR spectra of their $(R)$ - and $(S)$ - $O$-acetylmandelate esters (Scheme 3, example $\Delta(S-R)$ data are shown for compound $\mathbf{8 d}$ ).

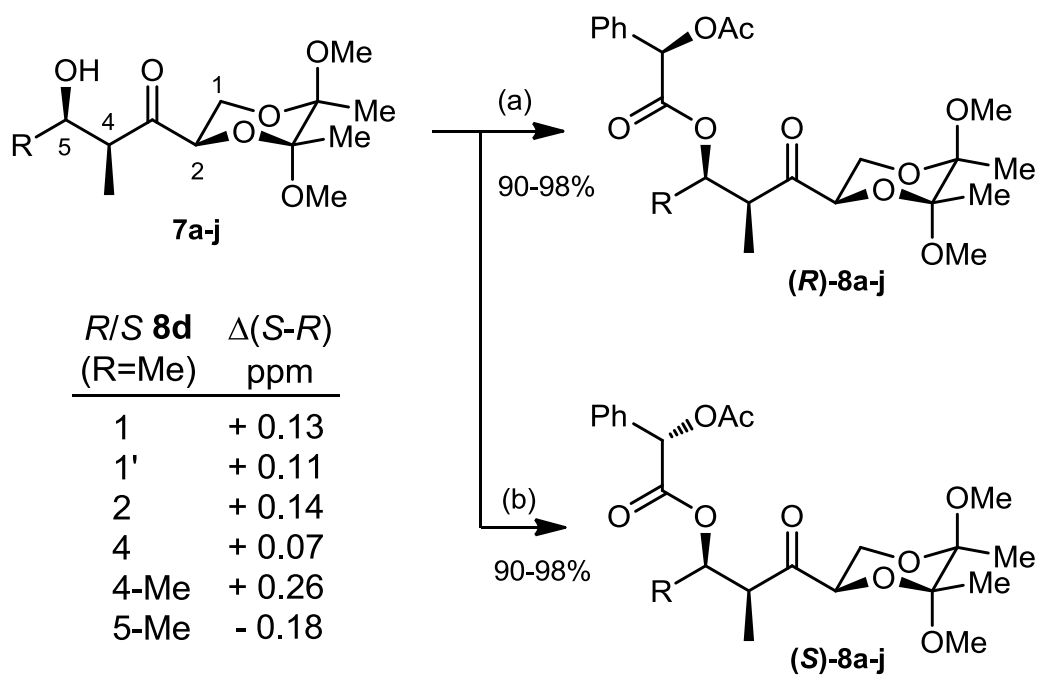

Scheme 3 Reagents and conditions: (a) $(R)$ - $O$-acetylmandelic acid, EDCI, DMAP, DCM, 0 ${ }^{\circ} \mathrm{C}$; (b) (S)-O-acetylmandelic acid, EDCI, DMAP, DCM, $0{ }^{\circ} \mathrm{C}$.

The stereochemical outcome of the reaction can be explained on the basis of a preferred enolate conformation in which the enolate $\mathrm{C}-\mathrm{O}$ bond and the $\alpha^{\prime} \mathrm{C}-\mathrm{O}$ bond are aligned in an antiperiplanar orientation for dipolar reasons. The approach of the aldehyde towards the least hindered face of the enolate in a Zimmerman-Traxler transition state then leads to the observed diastereoselectivity (Scheme 4). ${ }^{3 \mathrm{k}, 11}$ 


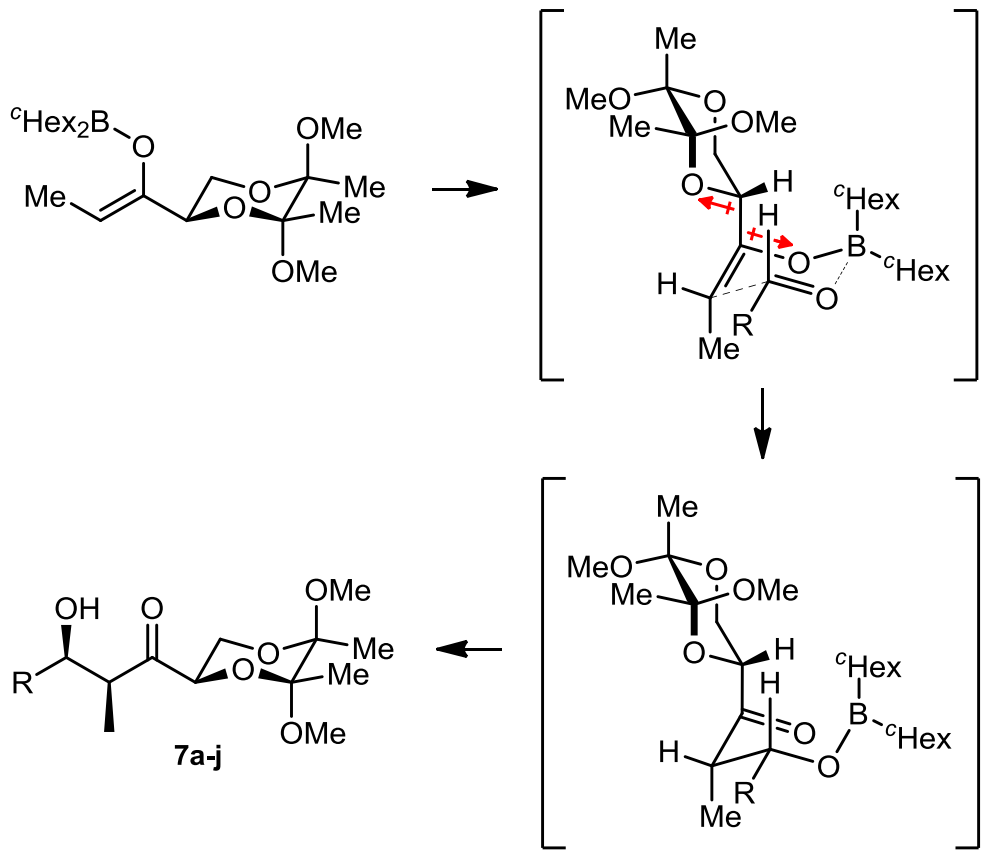

Scheme 4. Possible transition state model for the aldol addition. Dipoles shown in red.

$p$-Bromobenzaldehyde $\mathbf{6 b}$ was specifically chosen in the hope that the $p$-bromobenzene ring might impart crystallinity to the corresponding product $\mathbf{7 b}$. Pleasingly, this turned out to be the case and an X-ray crystal structure was obtained (Figure 1). In addition to providing further evidence for the relative syn-aldol stereochemistry, the presence of the heavy bromine atom also allowed the absolute stereochemistry to be determined from the X-ray data (which was in full agreement with the $O$-acetylmandelate analysis).



Figure 1. X-ray structure of $\mathbf{7 b}$. Thermal ellipsoids are shown at 50\%. 
In addition to the use of dicyclohexylboron chloride, we also briefly explored titanium reagents and found titanium tetrachloride to be equally competent in effecting these transformations (affording the same diastereomer of product) using diisopropylethylamine as the base and dichloromethane as the solvent (Table 1, entries 2,4,6). Conveniently, commercial grade material (purchased as a $1 \mathrm{M}$ solution in dichloromethane) was found to be adequate, providing a possible advantage over the use of dicyclohexylboron chloride. As the titanium derived by-products are all easily hydrolysed and extracted into the aqueous phase on workup, this is also a potential advantage over the boron reagent (which leads to cyclohexyl byproducts whose removal generally necessitates additional workup steps). As the observed stereochemical outcome of the titanium mediated reaction was the same as the boron mediated aldol addition, it is also possible that the transition-state is similar to that shown in Scheme 4. Unlike the boron atom in dialkylboron enolates, the titanium metal in the enolate can bind to additional Lewis basic groups, such as the $\alpha^{\prime}$-alkoxy group in 4 . This might be expected to lead to the alternative syn aldol stereochemistry via an alternative enolate conformation. However, it is possible that the 2,3-butane-diacetal moiety (containing two adjacent quaternary carbons) would cause too much steric crowding to permit such a chelated structure (Figure 2). This might perhaps lead to a similar 'dipole aligned' Zimmerman-Traxler transition state as suggested for the boron mediated reaction.
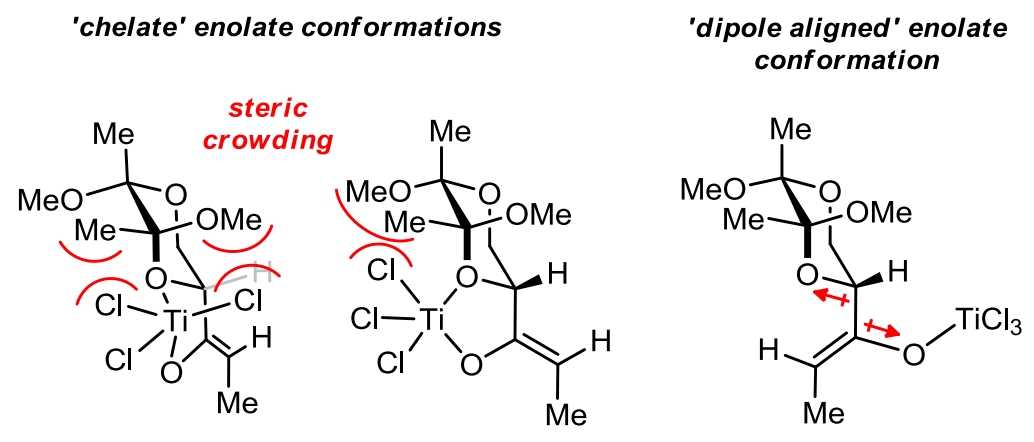

Figure 2. Potential steric crowding in hypothetical chelated titanium enolate conformations (left) and proposed favoured dipole-aligned enolate (right).

Having established the efficacy of the aldol coupling, we wanted to establish that the 2,3butane-diacetal group could be removed in order to reveal the keto-diol functionality. Perhaps unsurprisingly, when this was attempted using trifluoroacetic acid (a reagent we have commonly used for this transformation), the reaction was also accompanied by a rapid retroaldol cleavage (Scheme 5). We also found that the retro-aldol cleavage of $7 \mathbf{a}$ was rapid at 
room temperature using methanolic potassium carbonate, leading to the aldehyde and ketone in quantitative yield (Scheme 5). As yet, we have been unable to determine the order in which the butane-diacetal cleavage and retro-aldol processes occur under acidic conditions.<smiles>CCC(=O)[C@H](O)COc1ccccc1</smiles>

Scheme 5. Retro-aldol cleavage transformations of 7a.

To prevent the possibility of the retro-aldol process, we acylated the secondary alcohol in 7a. With this change, the butane-diacetal cleavage of the ester $\mathbf{1 0}$ was able to be performed in high yield (Scheme 6). We found that a THF-water solvent mixture, which provided a homogeneous reaction mixture after addition of TFA, led to the highest yield (95\%) for this reaction.<smiles>COC1(OC)OCC(C(=O)C(C)[C@H](O)c2ccccc2)C1(C)OC</smiles>

$7 \mathrm{a}$<smiles>CC(=O)O[C@H](c1ccccc1)[C@H](C)C(=O)O</smiles><smiles>[Y20]C(C)(C)O[Na]</smiles>

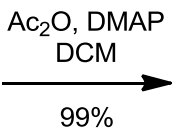

$99 \%$

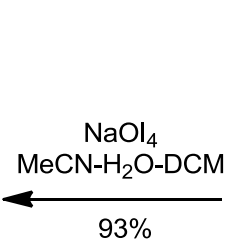

$93 \%$<smiles>COC1(OC)OC2CC(C(=O)C(C)C(OC(C)=O)c3ccccc3)C1(C)O2</smiles>

10

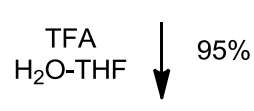<smiles>C[C@H](C(=O)[C@H](O)CO)[C@@H](OC(=O)O)c1ccccc1</smiles>

11<smiles>C[C@H](C(=O)O)[C@@H](O)c1ccccc1</smiles>

13

Scheme 6. Butane-diacetal cleavage and conversion to known hydroxy acid $\mathbf{1 3}$. 
The keto-triol moiety in $\mathbf{1 1}$ could be cleaved to the carboxylic acid $\mathbf{1 2}$ in $93 \%$ yield using sodium periodate in $\mathrm{MeCN}-\mathrm{H}_{2} \mathrm{O}-\mathrm{DCM}$. Saponification of the acetate group then afforded the well known hydroxy acid 13. Spectroscopic data and optical rotation values for this compound matched those provided in the literature, ${ }^{12}$ further confirming the diastereoselectivity of the aldol addition.

\section{Conclusion}

In conclusion, we have demonstrated that boron and titanium mediated aldol reactions of the mannitol derived 2,3-butane-diacetal ethyl ketone 4 proceed with high levels of substrate directed stereocontrol. The relative syn aldol stereochemistry was determined by analysis of the $J$-coupling values between the $\alpha$ and $\beta$ hydrogens in the ${ }^{1} \mathrm{H}$ NMR spectra. The absolute sense of stereocontrol was determined using X-ray crystallography, by NMR analysis of acetylmandelate derivatives and by conversion of aldol product $7 \mathbf{a}$ to the known hydroxy acid 13. We are currently investigating the use of this reaction in the synthesis of several polyketide natural products.

\section{Experimental Section}

\section{General Experimental Details:}

Unless otherwise stated, reactions were performed using glassware that was dried by heating with a heat-gun under a flow of argon. Argon gas was pre-dried by passing through a cartridge of granular calcium chloride. Unless otherwise stated, all reagents were used as supplied from commercial sources without further purification. Dichloromethane used in reactions was purified using continuously recycling distillation over calcium hydride under a dry argon atmosphere. Tetrahydrofuran and diethyl ether used in reactions were pre-dried over freshly pressed sodium wire and then further purified using continuously recycling distillation over a mixture of calcium hydride and lithium aluminium hydride under a dry argon atmosphere using triphenylmethane as an indicator. Triethylamine and diisopropylethylamine were purified by distillation over calcium hydride and were stored over calcium hydride under an atmosphere of dry argon. Distilled water and aqueous solutions were prepared on site. Flash column chromatography was performed using Merck 9385 grade silica gel purchased from Sigma-Aldrich. Analytical thin layer chromatography was performed using Merck Silica gel 60 F254 $1 \mathrm{~mm}$ glass plates and visualised either by 
ultraviolet radiation $(254 \mathrm{~nm})$ or by staining with potassium permanganate, cerium ammonium molybdate or vanillin solutions prepared by known procedures. 40/60 Petroleum ether refers to the distillate collected between $40-60{ }^{\circ} \mathrm{C}$. ${ }^{1} \mathrm{H}$ spectra were recorded on Bruker Avance 300 (300 MHz) or Bruker Avance DPX-400 (400 MHz) spectrometers. Chemical shifts are reported in parts per million ( $\mathrm{ppm}$ ) and the spectra are calibrated to the residual protic form of the deuterated solvent (7.26 ppm $\mathrm{CDCl}_{3} ; 3.31 \mathrm{ppm} \mathrm{CD}_{3} \mathrm{OD}$ ). The multiplicity of a signal is reported as: $\mathrm{s}$ - singlet; $\mathrm{d}$ - doublet; $\mathrm{t}$ - triplet; $\mathrm{br}$ - broad; $\mathrm{m}$ - multiplet. Coupling constants $(J)$ are given in hertz $(\mathrm{Hz})$ to 1 decimal place. Apodisation functions (Gaussian, exponential, sine bell) were used to resolve multiplets in some cases. The centre of each peak is reported with the exception of unresolved multiplet signals where a range of ppm values are given. ${ }^{13} \mathrm{C}$ NMR spectra were recorded on Bruker Avance $300(75 \mathrm{MHz})$ or Bruker Avance DPX-400 (100 MHz) spectrometers with complete proton decoupling. Chemical shifts are reported in ppm and the spectra are calibrated to the deuterated solvent as the internal deuterium lock (77.00 ppm $\left.\mathrm{CDCl}_{3} ; 49.00 \mathrm{ppm} \mathrm{CD}_{3} \mathrm{OD}\right) .{ }^{13} \mathrm{C}$ signals are singlets unless otherwise stated and are reported to 2 decimal places. Infrared Spectroscopy was carried out using a thin film (evaporated from solution) on a Thermo Scientific Nicolet iS10 ATR spectrometer. Mass Spectrometry was carried out on a Waters LCMS with electrospray ionisation and a QTOF analyser.

\section{Synthesis and Spectroscopic Data:}

1-((2R,5R,6R)-5,6-dimethoxy-5,6-dimethyl-1,4-dioxan-2-yl)propan-1-one 4.

Step 1) Grignard addition: To a stirring solution of the starting aldehyde $\mathbf{3}$ (10.0 g, 49.0 mmol, 1.0 equiv) in dry tetrahydrofuran $(300 \mathrm{~mL})$ was added dropwise a solution of ethylmagnesium bromide $\left(2.5 \mathrm{M}\right.$ in $\mathrm{Et}_{2} \mathrm{O}, 98.0 \mathrm{~mL}, 98.0 \mathrm{mmol}, 2.0$ equiv) at $-78{ }^{\circ} \mathrm{C}$ (acetone/dry-ice bath) under an atmosphere of dry argon. The reaction mixture was stirred at this temperature for $1 \mathrm{hr}$ after which time it was quenched by the addition of saturated aqueous ammonium chloride $(60 \mathrm{~mL})$ followed by water $(100 \mathrm{~mL})$ and diethyl ether $(200$ $\mathrm{mL}$ ) with rapid stirring. The reaction mixture was allowed to warm to room temperature and the layers were separated. The aqueous layer was extracted thrice with diethyl ether $(3 \times 60$ $\mathrm{mL})$ and the combined organic layers were washed with water $(100 \mathrm{~mL})$, brine $(100 \mathrm{~mL})$ and dried over magnesium sulfate. The solvent was removed under reduced pressure to afford a 
crude material that was used in the next reaction without further purification or characterisation.

Step 2) Swern oxidation: A solution of dimethyl sulfoxide (10.44 mL, $147 \mathrm{mmol}, 3.0$ equiv) in dichloromethane $(200 \mathrm{~mL})$ was added dropwise to a stirring solution of oxalyl chloride (6.30 mL, $73.5 \mathrm{mmol}, 1.5$ equiv) in dichloromethane $(200 \mathrm{~mL})$ at $-78{ }^{\circ} \mathrm{C}$ under an atmosphere of dry argon. The reaction was stirred at this temperature for $20 \mathrm{~min}$. A solution of the crude material from the Grignard addition step in dichloromethane (200 mL) was added dropwise at $-78^{\circ} \mathrm{C}$. The reaction mixture was stirred at this temperature for $20 \mathrm{~min}$. Triethylamine (40.9 mL, $294 \mathrm{mmol}, 6.0$ equiv) was added dropwise at $-78{ }^{\circ} \mathrm{C}$. The reaction mixture was stirred at this temperature for $15 \mathrm{~min}$ before warming to $-15^{\circ} \mathrm{C}$ (acetone/ice bath) and stirring for a further $45 \mathrm{~min}$. The reaction was quenched by the addition of saturated aqueous ammonium chloride $(100 \mathrm{~mL})$ followed by diethyl ether $(600 \mathrm{~mL})$. The mixture was allowed to warm to room temperature and the layers were separated. The aqueous layer was extracted thrice with diethyl ether $(3 \times 100 \mathrm{~mL})$. The combined organic layers were washed with saturated ammonium chloride solution $(200 \mathrm{~mL})$, water $(200 \mathrm{~mL})$ and brine $(200 \mathrm{~mL})$ before drying over magnesium sulfate. The solvent was removed under reduced pressure and to afford a crude product which was purified by column chromatography on silica gel (Merck 9385 grade) eluting with a gradient from 40/60 petroleum ether to a 1:1 mixture of 40/60 petroleum ether to diethyl ether. After removal of solvent under reduced pressure, the product was isolated as a colourless oil (10.57 g, 93\% over two steps).

${ }^{1}$ H NMR $\left(400 \mathrm{MHz}, \mathrm{CDCl}_{3}\right) \delta 4.34(\mathrm{dd}, J=10.5,4.3 \mathrm{~Hz}, 1 \mathrm{H}), 3.73(\mathrm{dd}, J=11.5,4.3 \mathrm{~Hz}$, $1 \mathrm{H}), 3.68(\mathrm{dd}, J=11.5,10.5 \mathrm{~Hz}, 1 \mathrm{H}), 3.31(\mathrm{~s}, 3 \mathrm{H}), 3.25$ (s, 3H), $2.78(\mathrm{dq}, J=18.8,7.2 \mathrm{~Hz}$, $1 \mathrm{H}), 2.56(\mathrm{dq}, J=18.8,7.2 \mathrm{~Hz}, 1 \mathrm{H}), 1.35$ (s, 3H), 1.28 (s, 3H), 1.03 (dd, $J=7.2,7.2 \mathrm{~Hz}, 3 \mathrm{H})$. ${ }^{13}$ C NMR (101 MHz, $\left.\mathrm{CDCl}_{3}\right) \delta$ 209.3, 99.7, 98.1, 72.6, 59.7, 48.3, 48.1, 31.7, 17.7, 17.5, 6.7. FTIR (thin film) $v_{\max } \mathrm{cm}^{-1} 2990,2946,2833,1716,1459,1405,1374,1258,1212,1165$, 1137, 1115, 1054, 1035, 1010, 987, 944, 878, 841, 773. HRMS (ESI-TOF, m/z) calculated for $\mathrm{C}_{11} \mathrm{H}_{20} \mathrm{NaO}_{5}^{+}\left([\mathrm{M}+\mathrm{Na}]^{+}\right):$255.1203. Observed: 255.1213.

\section{General procedure for boron mediated aldol reactions:}


Triethylamine (179 $\mu \mathrm{L}, \mathrm{mmol}, 1.29 \mathrm{mmol}, 3.0$ equiv) was added dropwise to a solution of dicyclohexylboron chloride ( $188 \mu \mathrm{L}, 0.862 \mathrm{mmol}, 2.0$ equiv) in dry diethyl ether $(1 \mathrm{~mL})$ at $60{ }^{\circ} \mathrm{C}$ (chloroform/dry-ice bath) under a dry argon atmosphere. After stirring at this temperature for $2 \mathrm{~min}$, a solution of the starting ketone 4 (100 mg, $0.431 \mathrm{mmol}, 1.0$ equiv) in dry diethyl ether $(1 \mathrm{~mL})$ was added at $-60^{\circ} \mathrm{C}$. The mixture was stirred at this temperature for 30 min, during which a significant amount of white precipitate was formed. To this suspension was added a solution of the aldehyde (1.94 mmol, 4.5 equiv) in dry diethyl ether $(1 \mathrm{~mL})$. The mixture was stirred at $-60{ }^{\circ} \mathrm{C}$ for $5 \mathrm{~h}$. To the reaction mixture was added saturated aqueous ammonium chloride solution $(2 \mathrm{~mL})$ with rapid stirring followed by the addition of diethyl ether $(10 \mathrm{~mL})$. The mixture was allowed to warm to room temperature and the layers were separated. The aqueous layer was thrice extracted with diethyl ether $(3 \times 5$ $\mathrm{mL}$ ) and the combined organic layers were dried over magnesium sulfate before removing the solvent under reduced pressure. The organic material was then dissolved in methanol (6 $\mathrm{mL})$ and $\mathrm{pH} 7$ aqueous phosphate buffer $(2 \mathrm{~mL})$ was added to the resulting solution. Into this cloudy suspension was added aqueous hydrogen peroxide $(2 \mathrm{~mL}, 30 \% \mathrm{wt} / \mathrm{vol}$.) and the mixture was stirred rapidly at room temperature for $30 \mathrm{~min}$. Diethyl ether $(20 \mathrm{~mL})$ and water $(20 \mathrm{~mL})$ were added and the mixture was stirred rapidly for $2 \mathrm{~min}$. The layers were separated and the aqueous layer was extracted with diethyl ether $(10 \mathrm{~mL})$. The combined organic layers were washed with water $(10 \mathrm{~mL})$ before drying over magnesium sulfate. The solvent was removed under reduced pressure and the crude material was purified by column chromatography on silica gel (Merck 9385 grade), eluting with a solvent gradient from 40/60 petroleum ether to a 1:1 mixture of $40 / 60$ petroleum ether to diethyl ether.

\section{General procedure for titanium mediated aldol reactions:}

Diisopropylethylamine ( $85 \mu \mathrm{L}, 0.517 \mathrm{mmol}, 1.2$ equiv) was added to a solution of the starting ketone 4 (100 mg, $0.431 \mathrm{mmol}, 1.0$ equiv) in dichloromethane $(4 \mathrm{~mL})$ at $-78{ }^{\circ} \mathrm{C}$ (acetone/dryice bath) under a dry argon atmosphere. To this solution was added dropwise a solution of titanium tetrachloride in dichloromethane $\left(1.0 \mathrm{M}, 517 \mu \mathrm{L}, 0.517 \mathrm{mmol}, 1.2\right.$ equiv) at $-78{ }^{\circ} \mathrm{C}$. The reaction mixture was stirred at this temperature for 2 min. A solution of the aldehyde (0.517 mmol, 1.2 equiv) in dichloromethane was added dropwise at $-78{ }^{\circ} \mathrm{C}$. The reaction mixture was stirred at this temperature for $10 \mathrm{~min}$. Saturated aqueous sodium bicarbonate (10 
$\mathrm{mL})$ was added dropwise with rapid stirring, followed by diethyl ether $(10 \mathrm{~mL})$. The mixture was allowed to warm to room temperature and extracted thrice with diethyl ether $(3 \times 10$ $\mathrm{mL})$. The combined organic layers were washed with water $(10 \mathrm{~mL})$, brine $(10 \mathrm{~mL})$ and dried over magnesium sulfate. The solvent was removed under reduced pressure to afford the crude material which was purified was purified by column chromatography on silica gel (Merck 9385 grade), eluting with a solvent gradient from $40 / 60$ petroleum ether to a 1:1 mixture of $40 / 60$ petroleum ether to diethyl ether.

(2S,3S)-1-((2R,5R,6R)-5,6-dimethoxy-5,6-dimethyl-1,4-dioxan-2-yl)-3-hydroxy-2-methyl-3phenylpropan-1-one 7a.

(Boron aldol: $143 \mathrm{mg}$ from $100 \mathrm{mg}$ of 4, 98\%. Titanium aldol: $139 \mathrm{mg}$ from $100 \mathrm{mg}$ of 4, $95 \%)$

${ }^{1}$ H NMR $\left(400 \mathrm{MHz}, \mathrm{CDCl}_{3}\right) \delta 7.39-7.25(\mathrm{~m}, 5 \mathrm{H}), 5.12(\mathrm{dd}, J=4.6,3.1 \mathrm{~Hz}, 1 \mathrm{H}), 4.32(\mathrm{dd}$, $J=10.1,4.8 \mathrm{~Hz}, 1 \mathrm{H}), 3.70-3.59(\mathrm{~m}, 2 \mathrm{H}), 3.47(\mathrm{qd}, J=7.0,4.6 \mathrm{~Hz}, 1 \mathrm{H}), 3.29(\mathrm{~s}, 3 \mathrm{H}), 3.25$ (s, 3H), $2.61(\mathrm{~d}, J=3.1 \mathrm{~Hz}, 1 \mathrm{H}), 1.38(\mathrm{~s}, 3 \mathrm{H}), 1.29(\mathrm{~s}, 3 \mathrm{H}), 1.12(\mathrm{~d}, J=7.0 \mathrm{~Hz}, 3 \mathrm{H}) .{ }^{13} \mathbf{C}$ NMR $\left(101 \mathrm{MHz}, \mathrm{CDCl}_{3}\right) \delta 212.0,141.9,128.3,127.6,126.0,99.8,98.0,73.4,72.4,59.6$, 48.8, 48.5, 48.1, 17.7, 17.5, 10.1. FTIR (thin film) $v_{\max } \mathrm{cm}^{-1} 3484$ (br), 2991, 2946, 2833, 1710, 1493, 1453, 1374, 1336, 1212, 1137, 1112, 1053, 1034, 944, 876, 840, 765, 748. HRMS (ESI-TOF, m/z) calculated for $\mathrm{C}_{18} \mathrm{H}_{26} \mathrm{NaO}_{6}{ }^{+}\left([\mathrm{M}+\mathrm{Na}]^{+}\right)$: 361.1622. Observed: 361.1626 .

(2S,3S)-3-(4-bromophenyl)-1-((2R,5R,6R)-5,6-dimethoxy-5,6-dimethyl-1,4-dioxan-2-yl)-3hydroxy-2-methylpropan-1-one $\mathbf{7 b}$.

(Boron aldol: $169 \mathrm{mg}$ from $100 \mathrm{mg}$ of 4, 94\%. Titanium aldol: $165 \mathrm{mg}$ from $100 \mathrm{mg}$ of 4, $92 \%)$.

${ }^{1} \mathbf{H}$ NMR $\left(400 \mathrm{MHz}, \mathrm{CDCl}_{3}\right) \delta 7.47(\mathrm{~d}, J=8.4 \mathrm{~Hz}, 2 \mathrm{H}), 7.21(\mathrm{~d}, J=8.4 \mathrm{~Hz}, 2 \mathrm{H}), 5.06(\mathrm{dd}, J$ $=4.4,2.9 \mathrm{~Hz}, 1 \mathrm{H}), 4.34(\mathrm{dd}, J=10.5,4.5 \mathrm{~Hz}, 1 \mathrm{H}), 3.70-3.60(\mathrm{~m}, 2 \mathrm{H}), 3.37(\mathrm{qd}, J=7.0,4.4$ $\mathrm{Hz}, 1 \mathrm{H}), 3.26$ (s, 3H), 3.23 (s, 3H), 2.73 (d, $J=2.9 \mathrm{~Hz}, 1 \mathrm{H}), 1.35$ (s, 3H), 1.28 (s, 3H), 1.08 $(\mathrm{d}, J=7.0 \mathrm{~Hz}, 3 \mathrm{H}) .{ }^{13} \mathbf{C}$ NMR $\left(101 \mathrm{MHz}, \mathrm{CDCl}_{3}\right) \delta 212.1,140.9,131.4,127.7,121.4,99.8$, 98.1, 72.7, 72.6, 59.6, 48.5, 48.5, 48.1, 17.7, 17.5, 9.8. FTIR (thin film) $v_{\max } \mathrm{cm}^{-1} 3470$ (br), 
2991, 2946, 2833, 1710, 1487, 1455, 1374, 1213, 1165, 1139, 1114, 1071, 1054, 1035, 1010, 986, 944, 878, 829, 789. HRMS (ESI-TOF, m/z) calculated for $\mathrm{C}_{18} \mathrm{H}_{25} \mathrm{BrKO}_{6}^{+}\left([\mathrm{M}+\mathrm{K}]^{+}\right)$: 455.0466. Observed: 455.0472.

(2S,3R)-1-((2R,5R,6R)-5,6-dimethoxy-5,6-dimethyl-1,4-dioxan-2-yl)-3-hydroxy-2,4dimethylpentan-1-one 7c.

(Boron aldol: $143 \mathrm{mg}$ from $100 \mathrm{mg}$ of 4, 98\%. Titanium aldol: $119 \mathrm{mg}$ from $100 \mathrm{mg}$ of $\mathbf{4}$, $91 \%)$

${ }^{1} \mathbf{H}$ NMR $\left(400 \mathrm{MHz}, \mathrm{CDCl}_{3}\right) \delta 4.49(\mathrm{dd}, J=10.6,4.4 \mathrm{~Hz}, 1 \mathrm{H}), 3.78-3.63(\mathrm{~m}, 2 \mathrm{H}), 3.61-$ $3.54(\mathrm{~m}, 1 \mathrm{H}), 3.38(\mathrm{qd}, J=7.0,2.9 \mathrm{~Hz}, 1 \mathrm{H}), 3.32(\mathrm{~s}, 3 \mathrm{H}), 3.24(\mathrm{~s}, 3 \mathrm{H}), 2.12$ (d, $J=3.9 \mathrm{~Hz}$, 1H), $1.66(\mathrm{~m}, 1 \mathrm{H}), 1.35(\mathrm{~s}, 3 \mathrm{H}), 1.28(\mathrm{~s}, 3 \mathrm{H}), 1.07$ (d, $J=7.0 \mathrm{~Hz}, 3 \mathrm{H}), 1.01$ (d, $J=6.6 \mathrm{~Hz}$, 3H), $0.90(\mathrm{~d}, J=6.7 \mathrm{~Hz}, 3 \mathrm{H}) .{ }^{13} \mathbf{C}$ NMR $\left(101 \mathrm{MHz}, \mathrm{CDCl}_{3}\right) \delta$ 212.5, 99.8, 98.0, 76.4, 71.7, 59.8, 48.6, 48.1 43.7, 31.2, 19.2, 19.1, 17.7, 17.5, 8.1. FTIR (thin film) $v_{\max } \mathrm{cm}^{-1} 3516$ (br), 2957, 2874, 2834, 1709, 1459, 1374, 1332, 1262, 1213, 1140, 1115, 1037, 1013, 945, 878. HRMS (ESI-TOF, $\mathrm{m} / \mathrm{z})$ calculated for $\mathrm{C}_{15} \mathrm{H}_{28} \mathrm{KO}_{6}{ }^{+}\left([\mathrm{M}+\mathrm{K}]^{+}\right)$: 343.1517. Observed: 343.1526 .

(2S,3R)-1-((2R,5R,6R)-5,6-dimethoxy-5,6-dimethyl-1,4-dioxan-2-yl)-3-hydroxy-2methylbutan-1-one 7d.

(Boron aldol: $110 \mathrm{mg}$ from $100 \mathrm{mg}$ of 4, 92\%).

${ }^{1} \mathbf{H}$ NMR $\left(400 \mathrm{MHz}, \mathrm{CDCl}_{3}\right) \delta 4.43(\mathrm{dd}, J=10.3,4.7 \mathrm{~Hz}, 1 \mathrm{H}), 4.10(\mathrm{qd}, J=6.3,3.6 \mathrm{~Hz}, 1 \mathrm{H})$, 3.75-3.60 (m, 2H), 3.30 (s, 3H), 3.24 (s, 3H), 3.13 (qd, $J=7.1,3.6 \mathrm{~Hz}, 1 \mathrm{H}), 2.51$ (br s, 1H), $1.35(\mathrm{~s}, 3 \mathrm{H}), 1.28(\mathrm{~s}, 3 \mathrm{H}), 1.16(\mathrm{~d}, J=6.3 \mathrm{~Hz}, 3 \mathrm{H}), 1.12(\mathrm{~d}, J=7.1 \mathrm{~Hz}, 3 \mathrm{H}) .{ }^{13} \mathbf{C}$ NMR $(101$ $\left.\mathrm{MHz}, \mathrm{CDCl}_{3}\right) \delta 212.7,99.8,98.0,72.5,67.5,59.7,48.5,48.1,47.1,20.1,17.7,17.5,9.6$. FTIR (thin film) $v_{\max } \mathrm{cm}^{-1} 3493$ (br), 2972, 2947, 2834, 1707, 1454, 1374, 1331, 1281, 1212 , 1137, 1113, 1055, 1034, 1004, 944, 911, 877, 841. HRMS (ESI-TOF, m/z) calculated for $\mathrm{C}_{13} \mathrm{H}_{24} \mathrm{NaO}_{6}^{+}\left([\mathrm{M}+\mathrm{Na}]^{+}\right): 299.1465$. Observed: 299.1474 . 
$(2 S, 3 R)-1-((2 R, 5 R, 6 R)-5,6$-dimethoxy-5,6-dimethyl-1,4-dioxan-2-yl)-3-hydroxy-2methylheptan-1-one 7e.

(Boron aldol: $133 \mathrm{mg}$ from $100 \mathrm{mg}$ of 4, 97\%).

${ }^{1}$ H NMR $\left(400 \mathrm{MHz}, \mathrm{CDCl}_{3}\right) \delta 4.46(\mathrm{dd}, J=10.5,4.5 \mathrm{~Hz}, 1 \mathrm{H}), 3.93(\mathrm{~s}, 1 \mathrm{H}), 3.72(\mathrm{dd}, J=$ 11.5, 4.5 Hz, 1H), 3.67 (dd, $J=11.5,10.5 \mathrm{~Hz}, 1 \mathrm{H}), 3.31(\mathrm{~s}, 3 \mathrm{H}), 3.24(\mathrm{~s}, 3 \mathrm{H}), 3.17$ (qd, $J=$ 7.1, 3.1 Hz, 1H), 2.31 (br s, 1H), 1.60-1.25 (m, 6H), 1.35 (s, 3H), 1.28 (s, 3H), 1.09 (d, $J=$ $7.1 \mathrm{~Hz}, 3 \mathrm{H}), 0.89$ (t, $J=7.0 \mathrm{~Hz}, 3 \mathrm{H}) .{ }^{13} \mathbf{C} \mathbf{N M R}\left(101 \mathrm{MHz}, \mathrm{CDCl}_{3}\right) \delta$ 212.6, 99.8, 98.0, 72.2, 71.1, 59.7, 48.5, 48.1, 46.0, 34.0, 28.2, 22.5, 17.7, 17.5, 14.0, 8.9. FTIR (thin film) $v_{\max } \mathrm{cm}^{-1}$ 3509 (br), 2935, 2834, 1708, 1456, 1374, 1212, 1165, 1137, 1114, 1036, 1008, 969, 944, 877, 842. HRMS (ESI-TOF, m/z) calculated for $\mathrm{C}_{16} \mathrm{H}_{30} \mathrm{NaO}_{6}{ }^{+}\left([\mathrm{M}+\mathrm{Na}]^{+}\right)$: 341.1935. Observed: 341.1952 .

(2S,3R)-1-((2R,5R,6R)-5,6-dimethoxy-5,6-dimethyl-1,4-dioxan-2-yl)-3-hydroxy-2methylpentan-1-one 7f.

(Boron aldol: $115 \mathrm{mg}$ from $100 \mathrm{mg}$ of 4, 92\%).

${ }^{1}$ H NMR $\left(400 \mathrm{MHz}, \mathrm{CDCl}_{3}\right) \delta 4.48(\mathrm{dd}, J=10.3,4.7 \mathrm{~Hz}, 1 \mathrm{H}), 3.86(\mathrm{ddd}, J=8.2,5.4,3.1$ $\mathrm{Hz}, 1 \mathrm{H}), 3.77-3.66(\mathrm{~m}, 2 \mathrm{H}), 3.31(\mathrm{~s}, 3 \mathrm{H}), 3.24(\mathrm{~s}, 3 \mathrm{H}), 3.25-3.19(\mathrm{~m}, 1 \mathrm{H}), 1.60-1.40$ (m, 2H), $1.37(\mathrm{~s}, 3 \mathrm{H}), 1.30(\mathrm{~s}, 3 \mathrm{H}), 1.12(\mathrm{~d}, J=7.1 \mathrm{~Hz}, 3 \mathrm{H}), 0.98(\mathrm{dd}, J=7.4,7.4 \mathrm{~Hz}, 3 \mathrm{H}) .{ }^{13} \mathrm{C}$ NMR $\left(101 \mathrm{MHz}, \mathrm{CDCl}_{3}\right) \delta 212.5,99.8,98.0,72.8,72.2,59.8,48.5,48.1,45.7,27.3,17.7$, 17.5, 10.4, 9.0. FTIR (thin film) $v_{\max } \mathrm{cm}^{-1} 3516$ (br), 2941, 2878, 2834, 1708, 1456, 1374, 1331, 1212, 1138, 1114, 1035, 1003, 974, 945, 878, 842. HRMS (ESI-TOF, m/z) calculated for $\mathrm{C}_{14} \mathrm{H}_{30} \mathrm{NO}_{6}{ }^{+}\left(\left[\mathrm{M}+\mathrm{NH}_{4}\right]^{+}\right):$308.2068. Observed: 308.2070.

(2S,3R)-3-cyclohexyl-1-((2R,5R,6R)-5,6-dimethoxy-5,6-dimethyl-1,4-dioxan-2-yl)-3hydroxy-2-methylpropan-1-one 7g.

(Boron aldol: $138 \mathrm{mg}$ from $100 \mathrm{mg}$ of 4, 93\%).

${ }^{1}$ H NMR $\left(400 \mathrm{MHz}, \mathrm{CDCl}_{3}\right) \delta 4.49(\mathrm{dd}, J=10.6,4.4 \mathrm{~Hz}, 1 \mathrm{H}), 3.75(\mathrm{dd}, J=11.5,4.4 \mathrm{~Hz}$, 1H), $3.69(\mathrm{dd}, J=11.5,10.6 \mathrm{~Hz}, 1 \mathrm{H}), 3.66(\mathrm{dd}, J=8.4,2.8 \mathrm{~Hz}, 1 \mathrm{H}), 3.38(\mathrm{qd}, J=7.0,2.8$ 
$\mathrm{Hz}, 1 \mathrm{H}), 3.33$ (s, 3H), 3.25 (s, 3H), $2.10-2.01(\mathrm{~m}, 2 \mathrm{H}), 1.80-1.71(\mathrm{~m}, 2 \mathrm{H}), 1.70-1.56(\mathrm{~m}$, $3 \mathrm{H}), 1.41-1.10(\mathrm{~m}, 3 \mathrm{H}), 1.36(\mathrm{~s}, 3 \mathrm{H}), 1.29$ (s, 3H), 1.06 (d, J=7.0 Hz, 3H), $1.01-0.89$ (m, 2H). ${ }^{13} \mathrm{C}$ NMR $\left(101 \mathrm{MHz}, \mathrm{CDCl}_{3}\right) \delta 212.6,99.8,98.0,75.2,71.7,59.8,48.6,48.1,43.3$, 40.6, 29.5, 29.2, 26.3, 26.0, 25.8, 17.7, 17.5, 8.2. FTIR (thin film) $v_{\max } \mathrm{cm}^{-1} 3517$ (br), 2991, 2925, 2851, 1709, 1449, 1374, 1212, 1141, 1115, 1057, 1038, 1011, 978, 945, 879. HRMS (ESI-TOF, m/z) calculated for $\mathrm{C}_{18} \mathrm{H}_{32} \mathrm{NaO}_{6}{ }^{+}\left([\mathrm{M}+\mathrm{Na}]^{+}\right)$: 367.2091. Observed: 367.2098.

$(2 S, 3 R, E)-1-((2 R, 5 R, 6 R)-5,6-d i m e t h o x y-5,6-d i m e t h y l-1,4-d i o x a n-2-y l)-3-h y d r o x y-2-$ methyldodec-6-en-1-one $\mathbf{7 h}$.

(Boron aldol: $157 \mathrm{mg}$ from $100 \mathrm{mg}$ of 4, 94\%).

${ }^{1} \mathbf{H}$ NMR $\left(400 \mathrm{MHz}, \mathrm{CDCl}_{3}\right) \delta 5.50-5.33(\mathrm{~m}, 2 \mathrm{H}), 4.45(\mathrm{dd}, J=10.2,4.6 \mathrm{~Hz}, 1 \mathrm{H}), 3.94$ (ddd, $J=7.7,3.5,3.5 \mathrm{~Hz}, 1 \mathrm{H}), 3.76-3.61(\mathrm{~m}, 2 \mathrm{H}), 3.31(\mathrm{~s}, 3 \mathrm{H}), 3.24(\mathrm{~s}, 3 \mathrm{H}), 3.17$ (qd, $J=$ 7.1, 3.5 Hz, 1H), 2.33 (br s, 1H), $2.21-2.04(\mathrm{~m}, 2 \mathrm{H}), 2.03-1.90(\mathrm{~m}, 2 \mathrm{H}), 1.56(\mathrm{~m}, 1 \mathrm{H}), 1.44$ $(\mathrm{m}, 1 \mathrm{H}), 1.38-1.18(\mathrm{~m}, 6 \mathrm{H}), 1.35(\mathrm{~s}, 3 \mathrm{H}), 1.28(\mathrm{~s}, 3 \mathrm{H}), 1.11(\mathrm{~d}, J=7.1 \mathrm{~Hz}, 3 \mathrm{H}), 0.87(\mathrm{t}, J=$ $6.7 \mathrm{~Hz}, 3 \mathrm{H}) .{ }^{13} \mathrm{C}$ NMR $\left(101 \mathrm{MHz}, \mathrm{CDCl}_{3}\right) \delta 212.4,131.4,129.2,99.8,98.0,72.3,70.8,59.8$, 48.5, 48.1, 46.2, 34.2, 32.5, 31.4, 29.2, 29.1, 22.5, 17.7, 17.5, 14.0, 9.3. FTIR (thin film) $v_{\max }$ $\mathrm{cm}^{-1} 3516$ (br), 2990, 2925, 2872, 2854, 1708, 1456, 1373, 1212, 1165, 1139, 1115, 1056, 1036, 968, 945, 878, 842. HRMS (ESI-TOF, m/z) calculated for $\mathrm{C}_{21} \mathrm{H}_{38} \mathrm{NaO}_{6}{ }^{+}\left([\mathrm{M}+\mathrm{Na}]^{+}\right)$: 409.2561. Observed: 409.2568 .

(2S,3R,E)-1-((2R,5R,6R)-5,6-dimethoxy-5,6-dimethyl-1,4-dioxan-2-yl)-3-hydroxy-2-methyl5-phenylpent-4-en-1-one 7i.

(Boron aldol: $149 \mathrm{mg}$ from $100 \mathrm{mg}$ of 4, 95\%).

${ }^{1}$ H NMR $\left(400 \mathrm{MHz}, \mathrm{CDCl}_{3}\right) \delta 7.43-7.23(\mathrm{~m}, 5 \mathrm{H}), 6.65(\mathrm{~d}, J=15.9 \mathrm{~Hz}, 1 \mathrm{H}), 6.19(\mathrm{dd}, J=$ 15.9, 6.1 Hz, 1H), $4.64(\mathrm{~m}, 1 \mathrm{H}), 4.49$ (dd, $J=10.5,4.5 \mathrm{~Hz}, 1 \mathrm{H}), 3.76(\mathrm{dd}, J=11.5,4.5 \mathrm{~Hz}$, $1 \mathrm{H}), 3.71(\mathrm{dd}, J=11.5,10.5 \mathrm{~Hz}, 1 \mathrm{H}), 3.40$ (qd, $J=7.0,4.3 \mathrm{~Hz}, 1 \mathrm{H}), 3.33$ (s, 3H), 3.26 (s, 3H), $2.64(\mathrm{~s}, 1 \mathrm{H}), 1.39(\mathrm{~s}, 3 \mathrm{H}), 1.31(\mathrm{~s}, 3 \mathrm{H}), 1.20(\mathrm{~d}, J=7.0 \mathrm{~Hz}, 3 \mathrm{H}) .{ }^{13} \mathbf{C} \mathbf{N M R}(101 \mathrm{MHz}$, $\left.\mathrm{CDCl}_{3}\right) \delta 211.8,136.5,131.5,128.9,128.6,127.8,126.5,99.9,98.1,72.8,72.7,59.7,48.5$, 48.1, 46.8, 17.7, 17.5, 10.5. FTIR (thin film) $v_{\max } \mathrm{cm}^{-1} 3479$ (br), 2991, 2945, 2833, 1709, 
1494, 1449, 1374, 1332, 1212, 1139, 1114, 1054, 1034, 966, 944, 877, 841, 747. HRMS

(ESI-TOF, m/z) calculated for $\mathrm{C}_{20} \mathrm{H}_{28} \mathrm{NaO}_{6}{ }^{+}\left([\mathrm{M}+\mathrm{Na}]^{+}\right)$: 387.1778. Observed: 387.1790.

(2S,3R,E)-1-((2R,5R,6R)-5,6-dimethoxy-5,6-dimethyl-1,4-dioxan-2-yl)-3-hydroxy-2methyloct-4-en-1-one 7j.

(Boron aldol: $138 \mathrm{mg}$ from $100 \mathrm{mg}$ of 4, 97\%).

${ }^{1} \mathbf{H}$ NMR $\left(400 \mathrm{MHz}, \mathrm{CDCl}_{3}\right) \delta 6.01(\mathrm{dt}, J=15.4,6.8,1 \mathrm{H}), 5.75(\mathrm{dd}, J=15.4,6.5 \mathrm{~Hz}, 1 \mathrm{H})$, $4.76(\mathrm{dd}, J=10.5,4.4 \mathrm{~Hz}, 1 \mathrm{H}), 4.70(\mathrm{~m}, 1 \mathrm{H}), 4.05(\mathrm{dd}, J=11.5,4.3 \mathrm{~Hz}, 1 \mathrm{H}), 4.00(\mathrm{dd}, J=$ 11.5, $10.5 \mathrm{~Hz}, 1 \mathrm{H}), 3.66$ (s, 3H), $3.64-3.55$ (m, 1H), 3.58 (s, 3H), 2.72 (s, 1H), $2.39-2.28$ (m, 2H), $1.77-1.70(\mathrm{~m}, 2 \mathrm{H}), 1.69(\mathrm{~s}, 3 \mathrm{H}), 1.62(\mathrm{~s}, 3 \mathrm{H}), 1.43(\mathrm{~d}, J=7.0 \mathrm{~Hz}, 3 \mathrm{H}), 1.22$ (t, $J=$ $7.3 \mathrm{~Hz}, 3 \mathrm{H}) .{ }^{13} \mathrm{C}$ NMR $\left(101 \mathrm{MHz}, \mathrm{CDCl}_{3}\right) \delta 211.7,133.4,129.4,99.8,98.0,73.0,72.5,59.7$, 48.5, 48.1, 46.7, 34.3, 22.2, 17.7, 17.5, 13.6, 10.5. FTIR (thin film) $v_{\max } \mathrm{cm}^{-1} 3485$ (br), 2956, 2932, 2873, 2834, 1709, 1456, 1374, 1212, 1165, 1138, 1114, 1055, 1035, 968, 945, 877, 841. HRMS (ESI-TOF, m/z) calculated for $\mathrm{C}_{17} \mathrm{H}_{34} \mathrm{NO}_{6}{ }^{+}\left(\left[\mathrm{M}+\mathrm{NH}_{4}\right]^{+}\right)$: 348.2381 . Observed: 348.2396.

\section{General procedure for the formation of $(R)$ - and $(S)$-acetylmandelates:}

A mixture of the starting hydroxy ketone $(0.015 \mathrm{mmol}, 1.0$ equiv), the corresponding $(R)$ or (S)-O-acetylmandelic acid (5.8 $\mathrm{mg}, 0.030 \mathrm{mmol}, 2.0$ equiv), $N$-(3-dimethylaminopropyl)- $N$ 'ethylcarbodiimide hydrochloride $(5.8 \mathrm{mg}, \quad 0.030 \mathrm{mmol}, 2.0$ equiv) and $\mathrm{N}, \mathrm{N}-4-$ dimethylaminopyridine $(3.7 \mathrm{mg}, 0.030 \mathrm{mmol}, 2.0$ equiv) in dry dichloromethane $(1 \mathrm{~mL})$ was stirred at room temperature for $2 \mathrm{~h}$. Diethyl ether $(3 \mathrm{~mL})$ and water $(3 \mathrm{~mL})$ were added and the mixture was stirred for $10 \mathrm{~min}$. The layers were separated and the organic layer was washed with saturated aqueous sodium bicarbonate $(1 \mathrm{~mL})$, saturated aqueous ammonium chloride $(1 \mathrm{~mL})$ then dried over magnesium sulfate. The solvent was removed under reduced pressure to afford the product as a viscous colourless oil.

$(R)-(1 S, 2 S)-3-((2 R, 5 R, 6 R)-5,6-d i m e t h o x y-5,6-d i m e t h y l-1,4-d i o x a n-2-y l)-2-m e t h y l-3-o x o-1-$ phenylpropyl 2-acetoxy-2-phenylacetate $(\boldsymbol{R})-\mathbf{8 a}$.

(7.3 $\mathrm{mg}$ from $5.0 \mathrm{mg}$ of $\mathbf{7 a}, 95 \%)$. 
${ }^{1}$ H NMR $\left(400 \mathrm{MHz}, \mathrm{CDCl}_{3}\right) \delta 7.50-7.38(\mathrm{~m}, 5 \mathrm{H}), 7.33-7.21(\mathrm{~m}, 5 \mathrm{H}), 6.32(\mathrm{~d}, J=5.3 \mathrm{~Hz}$, 1H), $5.96(\mathrm{~s}, 1 \mathrm{H}), 4.18(\mathrm{dd}, J=10.6,4.3 \mathrm{~Hz}, 1 \mathrm{H}), 3.59-3.43(\mathrm{~m}, 3 \mathrm{H}), 3.26(\mathrm{~s}, 3 \mathrm{H}), 3.20$ (s, 3H), 2.17 (s, 3H), $1.38(\mathrm{~s}, 3 \mathrm{H}), 1.26(\mathrm{~s}, 3 \mathrm{H}), 0.77(\mathrm{~d}, J=6.8 \mathrm{~Hz}, 3 \mathrm{H}) .{ }^{\mathbf{1 3}} \mathbf{C}$ NMR $(101 \mathrm{MHz}$, $\left.\mathrm{CDCl}_{3}\right) \delta 208.0,170.2,167.9,138.3,133.6,129.3,128.8,128.5,128.0,127.6,125.8,99.9$, 98.0, 76.0, 74.5, 72.0, 59.5, 48.7, 48.0, 47.9, 20.6, 17.8, 17.6, 9.5. FTIR (thin film) $v_{\max } \mathrm{cm}^{-1}$ 2991, 2947, 2834, 1745, 1716, 1497, 1455, 1373, 1227, 1168, 1141, 1114, 1053, 1036, 1011, 973, 943, 878, 737, 698. HRMS (ESI-TOF, m/z) calculated for $\mathrm{C}_{28} \mathrm{H}_{34} \mathrm{KO}_{9}{ }^{+}\left([\mathrm{M}+\mathrm{K}]^{+}\right)$: 553.1834. Observed: 553.1850.

$(S)-(1 S, 2 S)-3-((2 R, 5 R, 6 R)-5,6$-dimethoxy-5,6-dimethyl-1,4-dioxan-2-yl)-2-methyl-3-oxo-1phenylpropyl 2-acetoxy-2-phenylacetate $(\boldsymbol{S})$-8a.

(7.3 mg from 7a, 96\%).

${ }^{1} \mathbf{H}$ NMR $\left(400 \mathrm{MHz}, \mathrm{CDCl}_{3}\right) \delta 7.43-7.29(\mathrm{~m}, 5 \mathrm{H}), 7.19-7.10(\mathrm{~m}, 3 \mathrm{H}), 6.95-6.89(\mathrm{~m}, 2 \mathrm{H})$, $6.30(\mathrm{~d}, J=6.2 \mathrm{~Hz}, 1 \mathrm{H}), 5.98(\mathrm{~s}, 1 \mathrm{H}), 4.23(\mathrm{dd}, J=10.9,4.1 \mathrm{~Hz}, 1 \mathrm{H}), 3.65-3.58(\mathrm{~m}, 2 \mathrm{H})$, 3.52 (dd, $J=11.4,10.9 \mathrm{~Hz}, 1 \mathrm{H}), 3.23$ (s, 3H), 3.21 (s, 3H), 2.18 (s, 3H), 1.38 (s, 3H), 1.26 (s, $3 \mathrm{H}), 1.06(\mathrm{~d}, J=6.8 \mathrm{~Hz}, 3 \mathrm{H}) .{ }^{13} \mathbf{C}$ NMR $\left(101 \mathrm{MHz}, \mathrm{CDCl}_{3}\right) \delta 208.3,170.0,167.7,138.1$, 133.5, 129.2, 128.7, 128.2, 127.9, 127.8, 125.9, 99.9, 98.0, 76.2, 74.3, 72.2, 59.5, 48.6, 48.0, 47.9, 20.6, 17.8, 17.5, 10.6. FTIR (thin film) $v_{\max } \mathrm{cm}^{-1} 2991,2947,2834,1747,1717,1497$, 1455, 1373, 1229, 1206, 1170, 1142, 1115, 1054, 1036, 973, 944, 879, 749, 699. HRMS (ESI-TOF, m/z) calculated for $\mathrm{C}_{28} \mathrm{H}_{34} \mathrm{KO}_{9}{ }^{+}\left([\mathrm{M}+\mathrm{K}]^{+}\right)$: 553.1834. Observed: 553.1855.

(R)-(1S,2S)-1-(4-bromophenyl)-3-((2R,5R,6R)-5,6-dimethoxy-5,6-dimethyl-1,4-dioxan-2-yl)2-methyl-3-oxopropyl 2-acetoxy-2-phenylacetate $(\boldsymbol{R})-\mathbf{8 b}$.

(7.9 mg from $6.0 \mathrm{mg} \mathbf{7 b}, 93 \%$ ).

${ }^{1}$ H NMR $\left(400 \mathrm{MHz}, \mathrm{CDCl}_{3}\right) \delta 7.49-7.36(\mathrm{~m}, 7 \mathrm{H}), 7.12(\mathrm{~d}, J=8.4 \mathrm{~Hz}, 2 \mathrm{H}), 6.25(\mathrm{~d}, J=5.4$ $\mathrm{Hz}, 1 \mathrm{H}), 5.91(\mathrm{~s}, 1 \mathrm{H}), 4.16(\mathrm{dd}, J=10.7,4.2 \mathrm{~Hz}, 1 \mathrm{H}), 3.57-3.43(\mathrm{~m}, 3 \mathrm{H}), 3.24(\mathrm{~s}, 3 \mathrm{H}), 3.21$ (s, 3H), 2.17 (s, 3H), 1.37 (s, 3H), 1.26 (s, 3H), 0.78 (d, J = 6.8 Hz, 3H). ${ }^{13} \mathbf{C}$ NMR (101 $\left.\mathrm{MHz}, \mathrm{CDCl}_{3}\right) \delta 207.7,170.3,167.9,137.4,133.3,131.7,129.4,128.8,127.6,127.5,122.0$, 99.9, 98.0, 75.4, 74.6, 72.2, 59.5, 48.7, 48.1, 47.7, 20.6, 17.8, 17.6, 9.6. FTIR (thin film) $v_{\max }$ 
$\mathrm{cm}^{-1}$ 2990, 2947, 2834, 1744, 1716, 1620, 1489, 1455, 1373, 1228, 1207, 1167, 1141, 1114, 1074, 1053, 1035, 1010, 974, 942, 878. HRMS (ESI-TOF, m/z) calculated for $\mathrm{C}_{28} \mathrm{H}_{33} \mathrm{BrKO}_{9}{ }^{+}$ $\left([\mathrm{M}+\mathrm{K}]^{+}\right):$631.0940. Observed: 631.0954 .

(S)-(1S,2S)-1-(4-bromophenyl)-3-((2R,5R,6R)-5,6-dimethoxy-5,6-dimethyl-1,4-dioxan-2-yl)2-methyl-3-oxopropyl 2-acetoxy-2-phenylacetate $(\boldsymbol{S})-\mathbf{8 b}$.

(8.6 mg from $6.2 \mathrm{mg} \mathbf{7 b}, 97 \%)$.

${ }^{1}$ H NMR (400 MHz, $\left.\mathrm{CDCl}_{3}\right) \delta 7.44-7.32(\mathrm{~m}, 4 \mathrm{H}), 7.28-7.23(\mathrm{~m}, 3 \mathrm{H}), 6.77(\mathrm{~d}, J=8.4 \mathrm{~Hz}$, $2 \mathrm{H}), 6.23(\mathrm{~d}, J=6.3 \mathrm{~Hz}, 1 \mathrm{H}), 5.95(\mathrm{~s}, 1 \mathrm{H}), 4.25(\mathrm{dd}, J=10.9,4.0 \mathrm{~Hz}, 1 \mathrm{H}), 3.63(\mathrm{dd}, J=11.5$, $4.0 \mathrm{~Hz}, 1 \mathrm{H}), 3.57-3.49$ (m, 2H), 3.22 (s, 6H), 2.18 (s, 3H), 1.37 (s, 3H), 1.26 (s, 3H), 1.06 $(\mathrm{d}, J=6.8 \mathrm{~Hz}, 3 \mathrm{H}) .{ }^{13} \mathbf{C} \mathbf{N M R}\left(101 \mathrm{MHz}, \mathrm{CDCl}_{3}\right) \delta 208.1,170.1,167.7,137.3,133.4,131.4$, 129.4, 128.8, 127.8, 127.7, 122.0, 99.9, 98.1, 75.6, 74.2, 72.4, 59.5, 48.6, 48.1, 47.8, 20.6, 17.8, 17.5, 10.8. FTIR (thin film) $v_{\max } \mathrm{cm}^{-1} 2991,2947,2834,1746,1716,1489,1455,1373$, 1229, 1205, 1168, 1141, 1114, 1083, 1073, 1054, 1035, 1010, 973, 942, 878. HRMS (ESITOF, m/z) calculated for $\mathrm{C}_{28} \mathrm{H}_{33} \mathrm{BrKO}_{9}{ }^{+}\left([\mathrm{M}+\mathrm{K}]^{+}\right)$: 631.0940. Observed: 631.0957.

(R)-(2S,3R)-1-((2R,5R,6R)-5,6-dimethoxy-5,6-dimethyl-1,4-dioxan-2-yl)-2,4-dimethyl-1oxopentan-3-yl 2-acetoxy-2-phenylacetate $(\boldsymbol{R})$-8c.

(6.8 mg from $4.8 \mathrm{mg} 7 \mathrm{c}, 90 \%)$.

${ }^{1} \mathbf{H}$ NMR $\left(300 \mathrm{MHz}, \mathrm{CDCl}_{3}\right) \delta$ 7.41-7.39 (m, 5H), $5.83(\mathrm{~s}, 1 \mathrm{H}), 5.20(\mathrm{dd}, J=8.8,3.1 \mathrm{~Hz}$, $1 \mathrm{H}), 4.49$ (dd, $J=10.8,4.3 \mathrm{~Hz}, 1 \mathrm{H}), 3.58(\mathrm{dd}, J=11.5,4.3 \mathrm{~Hz}, 1 \mathrm{H}), 3.49$ (dd, $J=11.5,10.8$ $\mathrm{Hz}, 1 \mathrm{H}), 3.44-3.38$ (m, 4H), 3.20 (s, 3H), 2.18 (s, 3H), 1.97 - 1.81 (m, 1H), 1.33 (s, 3H), $1.27(\mathrm{~s}, 3 \mathrm{H}), 0.98(\mathrm{~d}, J=6.8 \mathrm{~Hz}, 3 \mathrm{H}), 0.93(\mathrm{~d}, J=6.6 \mathrm{~Hz}, 3 \mathrm{H}), 0.69(\mathrm{~d}, J=6.9 \mathrm{~Hz}, 3 \mathrm{H}) .{ }^{13} \mathbf{C}$ NMR $\left(75 \mathrm{MHz}, \mathrm{CDCl}_{3}\right) \delta 208.6,170.4,168.6,133.5,129.3,128.7,127.8,99.8,98.0,78.8$, 74.5, 71.3, 59.7, 48.9, 48.1, 42.9, 30.3, 20.7, 19.1, 18.8, 17.8, 17.6, 7.7. FTIR (thin film) $v_{\max }$ $\mathrm{cm}^{-1}$ 2964, 2947, 2878, 2834, 1742, 1716, 1497, 1456, 1372, 1329, 1231, 1209, 1140, 1177 , 1115, 1049, 1037, 1010, 971, 943, 879. HRMS (ESI-TOF, m/z) calculated for $\mathrm{C}_{25} \mathrm{H}_{36} \mathrm{KO}_{9}{ }^{+}$ $\left([\mathrm{M}+\mathrm{K}]^{+}\right): 519.1991$. Observed: 519.2003. 
$(S)-(2 S, 3 R)-1-((2 R, 5 R, 6 R)-5,6-d i m e t h o x y-5,6-d i m e t h y l-1,4-d i o x a n-2-y l)-2,4-d i m e t h y l-1-$ oxopentan-3-yl 2-acetoxy-2-phenylacetate $(\boldsymbol{S})$-8c.

(7.4 mg from $5.0 \mathrm{mg} \mathrm{7c,94 \% ).}$

${ }^{1}$ H NMR $\left(300 \mathrm{MHz}, \mathrm{CDCl}_{3}\right) \delta 7.47-7.34(\mathrm{~m}, 5 \mathrm{H}), 5.87(\mathrm{~s}, 1 \mathrm{H}), 5.21(\mathrm{dd}, J=7.6,4.5 \mathrm{~Hz}$, 1H), 4.55 (dd, $J=10.9,4.2 \mathrm{~Hz}, 1 \mathrm{H}), 3.74$ (dd, $J=11.3,4.2 \mathrm{~Hz}, 1 \mathrm{H}), 3.61$ (dd, $J=11.3,10.9$ Hz, 1H), 3.52 (qd, $J=6.9,4.5 \mathrm{~Hz}, 1 \mathrm{H}), 3.41(\mathrm{~s}, 3 \mathrm{H}), 3.24(\mathrm{~s}, 3 \mathrm{H}), 2.18(\mathrm{~s}, 3 \mathrm{H}), 1.72(\mathrm{~m}, 1 \mathrm{H})$, $1.35(\mathrm{~s}, 3 \mathrm{H}), 1.28(\mathrm{~s}, 3 \mathrm{H}), 1.04(\mathrm{~d}, J=6.9 \mathrm{~Hz}, 3 \mathrm{H}), 0.76(\mathrm{~d}, J=6.8 \mathrm{~Hz}, 3 \mathrm{H}), 0.53$ (d, $J=6.6$ $\mathrm{Hz}, 3 \mathrm{H}) .{ }^{13} \mathrm{C} \mathbf{N M R}\left(75 \mathrm{MHz}, \mathrm{CDCl}_{3}\right) \delta 209.1,170.0,168.6,134.0,129.2,128.7,127.7,99.8$, 98.0, 78.9, 74.3, 71.6, 59.8, 48.8, 48.1, 43.3, 30.2, 20.7, 19.1, 17.8, 17.7, 17.6, 9.3. FTIR (thin film) $v_{\max } \mathrm{cm}^{-1} 2963,2878,2834,1745,1716,1497,1456,1373,1332,1231,1210$, 1176, 1141, 1116, 1055, 1037, 1011, 968, 944, 879. HRMS (ESI-TOF, m/z) calculated for $\mathrm{C}_{25} \mathrm{H}_{36} \mathrm{KO}_{9}^{+}\left([\mathrm{M}+\mathrm{K}]^{+}\right): 519.1991$. Observed: 519.2008.

$(R)-(2 R, 3 S)-4-((2 R, 5 R, 6 R)-5,6$-dimethoxy-5,6-dimethyl-1,4-dioxan-2-yl)-3-methyl-4oxobutan-2-yl 2-acetoxy-2-phenylacetate $(\boldsymbol{R})-\mathbf{8 d}$.

(7.6 mg from $5.1 \mathrm{mg} \mathbf{7 d}, 91 \%)$.

${ }^{1} \mathbf{H}$ NMR $\left(300 \mathrm{MHz}, \mathrm{CDCl}_{3}\right) \delta 7.44-7.36(\mathrm{~m}, 5 \mathrm{H}), 5.80(\mathrm{~s}, 1 \mathrm{H}), 5.41(\mathrm{qd}, J=6.4,4.7 \mathrm{~Hz}$, $1 \mathrm{H}), 4.30(\mathrm{dd}, J=10.3,4.6 \mathrm{~Hz}, 1 \mathrm{H}), 3.59$ (dd, $J=11.3,4.6 \mathrm{~Hz}, 1 \mathrm{H}), 3.53(\mathrm{dd}, J=11.3,10.3$ $\mathrm{Hz}, 1 \mathrm{H}), 3.31$ (s, 3H), 3.21 (s, 3H), $3.23-3.13$ (m, 1H), 2.18 (s, 3H), 1.32 (s, 3H), 1.29 (d, J $=6.4 \mathrm{~Hz}, 3 \mathrm{H}), 1.26(\mathrm{~s}, 3 \mathrm{H}), 0.83(\mathrm{~d}, J=6.9 \mathrm{~Hz}, 3 \mathrm{H}) .{ }^{13} \mathbf{C ~ N M R}\left(101 \mathrm{MHz}, \mathrm{CDCl}_{3}\right) \delta 208.4$, 170.4, 168.3, 133.7, 129.3, 128.8, 127.6, 99.8, 98.0, 74.7, 71.8, 71.8, 59.7, 48.7, 48.0, 46.4, 20.6, 18.3, 17.7, 17.6, 9.3. FTIR (thin film) $v_{\max } \mathrm{cm}^{-1} 2989,2947,2834,1741,1715,1497$, 1455, 1373, 1324, 1264, 1229, 1210, 1177, 1138, 1114, 1082, 1054, 1032, 1003, 963, 943, 878. HRMS (ESI-TOF, m/z) calculated for $\mathrm{C}_{23} \mathrm{H}_{32} \mathrm{KO}_{9}{ }^{+}\left([\mathrm{M}+\mathrm{K}]^{+}\right): 491.1678$. Observed: 491.1693.

(S)-(2R,3S)-4-((2R,5R,6R)-5,6-dimethoxy-5,6-dimethyl-1,4-dioxan-2-yl)-3-methyl-4oxobutan-2-yl 2-acetoxy-2-phenylacetate $(\boldsymbol{S})-\mathbf{8 d}$.

(7.3 mg from $4.5 \mathrm{mg} \mathbf{7 d}, 99 \%)$. 
${ }^{1}$ H NMR $\left(300 \mathrm{MHz}, \mathrm{CDCl}_{3}\right) \delta 7.45-7.35(\mathrm{~m}, 5 \mathrm{H}), 5.80(\mathrm{~s}, 1 \mathrm{H}), 5.42(\mathrm{qd}, J=6.3,5.3 \mathrm{~Hz}$, $1 \mathrm{H}), 4.44(\mathrm{dd}, J=10.7,4.2 \mathrm{~Hz}, 1 \mathrm{H}), 3.72(\mathrm{dd}, J=11.5,4.2 \mathrm{~Hz}, 1 \mathrm{H}), 3.64(\mathrm{dd}, J=11.5,10.7$ $\mathrm{Hz}, 1 \mathrm{H}), 3.35-3.25$ (m, 4H), 3.24 (s, 3H), 2.18 (s, 3H), 1.34 (s, 3H), 1.28 (s, 3H), 1.11 (d, J $=3.2 \mathrm{~Hz}, 3 \mathrm{H}), 1.09(\mathrm{~d}, J=3.8 \mathrm{~Hz}, 3 \mathrm{H}) .{ }^{13} \mathbf{C} \mathbf{N M R}\left(75 \mathrm{MHz}, \mathrm{CDCl}_{3}\right) \delta 208.8,170.2,168.2$, 133.6, 129.2, 128.8, 127.5, 99.8, 98.0, 74.6, 72.0, 71.8, 59.7, 48.6, 48.1, 46.3, 20.6, 17.8, 17.7, 17.6, 10.4. FTIR (thin film) $v_{\max } \mathrm{cm}^{-1} 2989,2946,2834,1742,1715,1497,1455,1373$, 1327, 1264, 1229, 1209, 1177, 1138, 1113, 1082, 1053, 1032, 1003, 963, 943, 878. HRMS (ESI-TOF, m/z) calculated for $\mathrm{C}_{23} \mathrm{H}_{32} \mathrm{KO}_{9}{ }^{+}\left([\mathrm{M}+\mathrm{K}]^{+}\right)$: 491.1678. Observed: 491.1688 .

$(R)-(2 S, 3 R)-1-((2 R, 5 R, 6 R)-5,6$-dimethoxy-5,6-dimethyl-1,4-dioxan-2-yl)-2-methyl-1oxoheptan-3-yl 2-acetoxy-2-phenylacetate $(\boldsymbol{R})$-8e.

(7.8 mg from $5.3 \mathrm{mg} 7 \mathbf{7 e}, 94 \%)$.

${ }^{1}$ H NMR $\left(300 \mathrm{MHz}, \mathrm{CDCl}_{3}\right) \delta 7.42-7.36(\mathrm{~m}, 5 \mathrm{H}), 5.80$ (s, 1H), 5.43 (ddd, $J=7.5,6.4,3.5$ $\mathrm{Hz}, 1 \mathrm{H}), 4.37$ (dd, $J=10.6,4.4 \mathrm{~Hz}, 1 \mathrm{H}), 3.59$ (dd, $J=11.5,4.4 \mathrm{~Hz}, 1 \mathrm{H}), 3.51(\mathrm{dd}, J=11.5$, $10.6 \mathrm{~Hz}, 1 \mathrm{H}), 3.37$ (s, 3H), $3.30-3.23(\mathrm{~m}, 1 \mathrm{H}), 3.21(\mathrm{~s}, 3 \mathrm{H}), 2.18(\mathrm{~s}, 3 \mathrm{H}), 1.77-1.45$ (m, 2H), $1.33-1.20(\mathrm{~m}, 4 \mathrm{H}), 1.32(\mathrm{~s}, 3 \mathrm{H}), 1.26(\mathrm{~s}, 3 \mathrm{H}), 0.88(\mathrm{t}, J=6.9 \mathrm{~Hz}, 3 \mathrm{H}), 0.77$ (d, $J=6.9$ $\mathrm{Hz}, 3 \mathrm{H}) .{ }^{13} \mathrm{C}$ NMR $\left(101 \mathrm{MHz}, \mathrm{CDCl}_{3}\right) \delta 208.4,173.5,168.5,133.7,129.3,128.7,127.7$, 99.8, 98.0, 74.6, 74.5, 71.5, 59.7, 48.8, 48.1, 44.6, 32.0, 27.5, 22.2, 20.7, 17.7, 17.6, 13.9, 8.2. FTIR (thin film) $v_{\max } \mathrm{cm}^{-1} 2953,2873,2835,1742,1716,1497,1456,1373,1331,1263$, 1230, 1208, 1176, 1139, 1115, 1083, 1037, 1005, 967, 944, 879. HRMS (ESI-TOF, m/z) calculated for $\mathrm{C}_{26} \mathrm{H}_{38} \mathrm{KO}_{9}{ }^{+}\left([\mathrm{M}+\mathrm{K}]^{+}\right)$: 533.2147. Observed: 533.2170.

$(S)-(2 S, 3 R)-1-((2 R, 5 R, 6 R)-5,6-d i m e t h o x y-5,6-d i m e t h y l-1,4-d i o x a n-2-y l)-2-m e t h y l-1-$ oxoheptan-3-yl 2-acetoxy-2-phenylacetate $(\boldsymbol{S})$-8e.

(8.6 mg from $6.1 \mathrm{mg} 7 \mathbf{e}, 92 \%)$.

${ }^{1} \mathbf{H}$ NMR $\left(300 \mathrm{MHz}, \mathrm{CDCl}_{3}\right) \delta 7.46-7.33(\mathrm{~m}, 5 \mathrm{H}), 5.82(\mathrm{~s}, 1 \mathrm{H}), 5.40$ (ddd, $J=8.3,5.0 \mathrm{~Hz}$, $1 \mathrm{H}), 4.50(\mathrm{dd}, J=10.9,4.2 \mathrm{~Hz}, 1 \mathrm{H}), 3.73(\mathrm{dd}, J=11.5,4.2 \mathrm{~Hz}, 1 \mathrm{H}), 3.62(\mathrm{dd}, J=11.5,10.9$ $\mathrm{Hz}, 1 \mathrm{H}), 3.36$ (s, 3H), $3.34-3.29$ (m, 1H), 3.24 (s, 3H), 2.17 (s, 3H), $1.51-1.23(\mathrm{~m}, 2 \mathrm{H})$, $1.34(\mathrm{~s}, 3 \mathrm{H}), 1.28(\mathrm{~s}, 3 \mathrm{H}), 1.20-1.05(\mathrm{~m}, 2 \mathrm{H}), 1.06(\mathrm{~d}, J=6.9 \mathrm{~Hz}, 3 \mathrm{H}), 0.97-0.87(\mathrm{~m}, 2 \mathrm{H})$, 
$0.70(\mathrm{t}, J=7.3 \mathrm{~Hz}, 3 \mathrm{H}) .{ }^{13} \mathbf{C} \mathbf{N M R}\left(75 \mathrm{MHz}, \mathrm{CDCl}_{3}\right) \delta 208.9,170.1,168.6,133.8,129.2$, 128.7, 127.6, 99.8, 98.0, 74.5, 74.5, 71.8, 59.7, 48.7, 48.1, 45.1, 32.0, 27.1, 22.0, 20.7, 17.7, 17.6, 13.7, 9.6. FTIR (thin film) $v_{\max } \mathrm{cm}^{-1} 2954,2873,2834,1742,1713,1526,1496,1455$, 1373, 1332, 1230, 1208, 1176, 1140, 1115, 1083, 1037, 964, 944, 879. HRMS (ESI-TOF, $\mathrm{m} / \mathrm{z}$ ) calculated for $\mathrm{C}_{26} \mathrm{H}_{38} \mathrm{KO}_{9}{ }^{+}\left([\mathrm{M}+\mathrm{K}]^{+}\right): 533.2147$. Observed: 533.2170.

$(R)-(2 S, 3 R)-1-((2 R, 5 R, 6 R)-5,6$-dimethoxy-5,6-dimethyl-1,4-dioxan-2-yl)-2-methyl-1oxopentan-3-yl 2-acetoxy-2-phenylacetate $(\boldsymbol{R})-\mathbf{8 f}$.

(6.3 $\mathrm{mg}$ from $4.2 \mathrm{mg} \mathbf{7 f}, 93 \%)$.

${ }^{1} \mathbf{H}$ NMR $\left(300 \mathrm{MHz}, \mathrm{CDCl}_{3}\right) \delta 7.44-7.36(\mathrm{~m}, 5 \mathrm{H}), 5.81$ (s, 1H), 5.35 (ddd, $J=7.3,6.9,3.7$ $\mathrm{Hz}, 1 \mathrm{H}), 4.38(\mathrm{dd}, J=10.6,4.5 \mathrm{~Hz}, 1 \mathrm{H}), 3.59(\mathrm{dd}, J=11.5,4.5 \mathrm{~Hz}, 1 \mathrm{H}), 3.52(\mathrm{dd}, J=11.5$, $10.6 \mathrm{~Hz}, 1 \mathrm{H}), 3.37$ (s, 3H), $3.33-3.23(\mathrm{~m}, 1 \mathrm{H}), 3.21(\mathrm{~s}, 3 \mathrm{H}), 2.18(\mathrm{~s}, 3 \mathrm{H}), 1.78-1.52$ (m, 2H), $1.32(\mathrm{~s}, 3 \mathrm{H}), 1.26(\mathrm{~s}, 3 \mathrm{H}), 0.93(\mathrm{t}, J=7.4 \mathrm{~Hz}, 3 \mathrm{H}), 0.76(\mathrm{~d}, J=6.9 \mathrm{~Hz}, 3 \mathrm{H}) .{ }^{13} \mathbf{C ~ N M R}$ $\left(75 \mathrm{MHz}, \mathrm{CDCl}_{3}\right) \delta 208.5,170.4,168.5,133.5,129.3,128.7,127.7,99.8,98.0,75.8,74.6$, 71.4, 59.7, 48.8, 48.8, 44.2, 25.5, 20.7, 17.8, 17.6, 9.9, 8.1. FTIR (thin film) $v_{\max } \mathrm{cm}^{-1} 2946$, 2881, 2835, 1743, 1717, 1497, 1456, 1373, 1332, 1263, 1231, 1211, 1178, 1142, 1116, 1083, 1055, 1038, 1003, 970, 946, 879. HRMS (ESI-TOF, m/z) calculated for $\mathrm{C}_{24} \mathrm{H}_{34} \mathrm{KO}_{9}{ }^{+}$ $\left([\mathrm{M}+\mathrm{K}]^{+}\right): 505.1834$. Observed: 505.1843.

$(S)-(2 S, 3 R)-1-((2 R, 5 R, 6 R)-5,6-$ dimethoxy-5,6-dimethyl-1,4-dioxan-2-yl)-2-methyl-1oxopentan-3-yl 2-acetoxy-2-phenylacetate $(\boldsymbol{S})-\mathbf{8 f}$.

(7.3 mg from $4.8 \mathrm{mg} \mathbf{7 f}, 94 \%)$.

${ }^{1}$ H NMR $\left(300 \mathrm{MHz}, \mathrm{CDCl}_{3}\right) \delta 7.46-7.34(\mathrm{~m}, 5 \mathrm{H}), 5.82$ (s, 1H), 5.34 (ddd, $J=7.4,6.0,4.6$ $\mathrm{Hz}, 1 \mathrm{H}), 4.50$ (dd, $J=10.8,4.2 \mathrm{~Hz}, 1 \mathrm{H}), 3.73(\mathrm{dd}, J=11.5,4.2 \mathrm{~Hz}, 1 \mathrm{H}), 3.62(\mathrm{dd}, J=11.5$, $10.8 \mathrm{~Hz}, 1 \mathrm{H}), 3.41-3.33$ (m, 1H), 3.36 (s, 3H), 3.24 (s, 3H), 2.17 (s, 3H), 1.53 - 1.41 (m, 2H), $1.34(\mathrm{~s}, 3 \mathrm{H}), 1.28(\mathrm{~s}, 3 \mathrm{H}), 1.06(\mathrm{~d}, J=6.9 \mathrm{~Hz}, 3 \mathrm{H}), 0.63(\mathrm{t}, J=7.4 \mathrm{~Hz}, 3 \mathrm{H}) .{ }^{13} \mathbf{C ~ N M R}$ $\left(75 \mathrm{MHz}, \mathrm{CDCl}_{3}\right) \delta 208.9,170.1,168.6,133.8,129.2,128.7,127.6,99.8,98.0,76.0,74.5$, 71.7, 59.7, 48.7, 48.1, 44.5, 25.4, 20.7, 17.8, 17.6, 9.6, 9.4. FTIR (thin film) $v_{\max } \mathrm{cm}^{-1} 2945$, 2880, 2834, 1742, 1716, 1497, 1455, 1373, 1332, 1266, 1229, 1208, 1176, 1138, 1114, 1084, 
1054, 1035, 1004, 969, 944, 878. HRMS (ESI-TOF, m/z) calculated for $\mathrm{C}_{24} \mathrm{H}_{34} \mathrm{KO}_{9}{ }^{+}$ $\left([\mathrm{M}+\mathrm{K}]^{+}\right):$505.1834. Observed: 505.1848.

$(R)-(1 R, 2 S)-1-c y c l o h e x y l-3-((2 R, 5 R, 6 R)-5,6$-dimethoxy-5,6-dimethyl-1,4-dioxan-2-yl)-2methyl-3-oxopropyl 2-acetoxy-2-phenylacetate $(\boldsymbol{R})-\mathbf{8 g}$.

(7.6 mg from $5.2 \mathrm{mg} \mathbf{7 g}, 97 \%$ ).

${ }^{1}$ H NMR $\left(400 \mathrm{MHz}, \mathrm{CDCl}_{3}\right) \delta 7.42-7.36(\mathrm{~m}, 5 \mathrm{H}), 5.85(\mathrm{~s}, 1 \mathrm{H}), 5.27(\mathrm{dd}, J=8.6,3.1 \mathrm{~Hz}$, $1 \mathrm{H}), 4.48$ (dd, $J=10.8,4.2 \mathrm{~Hz}, 1 \mathrm{H}), 3.57$ (dd, $J=11.3,4.2 \mathrm{~Hz}, 1 \mathrm{H}), 3.49$ (dd, $J=11.3,10.8$ $\mathrm{Hz}, 1 \mathrm{H}), 3.44-3.38(\mathrm{~m}, 1 \mathrm{H}), 3.43$ (s, 3H), 3.20 (s, 3H), 2.17 (s, 3H), $1.82-1.62(\mathrm{~m}, 5 \mathrm{H})$, $1.33(\mathrm{~s}, 3 \mathrm{H}), 1.27(\mathrm{~s}, 3 \mathrm{H}), 1.26-0.84(\mathrm{~m}, 6 \mathrm{H}), 0.70(\mathrm{~d}, J=6.9 \mathrm{~Hz}, 3 \mathrm{H}) .{ }^{13} \mathbf{C}$ NMR (101 $\left.\mathrm{MHz}, \mathrm{CDCl}_{3}\right) \delta 208.6,170.3,168.6,133.7,129.3,128.6,127.8,99.8,98.0,77.9,74.5,71.3$, 59.7, 48.9, 48.1, 42.4, 39.6, 29.3, 29.1, 26.1, 25.9, 25.7, 20.7, 17.8, 17.6, 7.7. FTIR (thin film) $v_{\max } \mathrm{cm}^{-1} 2990,2928,2853,1743,1716,1451,1373,1319,1231,1210,1178,1141$, 1115, 1082, 1054, 1038, 1008, 975, 943， 890. HRMS (ESI-TOF, m/z) calculated for $\mathrm{C}_{28} \mathrm{H}_{40} \mathrm{KO}_{9}^{+}\left([\mathrm{M}+\mathrm{K}]^{+}\right): 559.2304$. Observed: 559.2321.

$(S)-(1 R, 2 S)-1$-cyclohexyl-3-((2R,5R,6R)-5,6-dimethoxy-5,6-dimethyl-1,4-dioxan-2-yl)-2methyl-3-oxopropyl 2-acetoxy-2-phenylacetate $(\boldsymbol{S})$-8g.

(8.0 mg from $5.6 \mathrm{mg} 7 \mathrm{~g}, \%)$.

${ }^{1} \mathbf{H}$ NMR $\left(400 \mathrm{MHz}, \mathrm{CDCl}_{3}\right) \delta 7.47-7.33(\mathrm{~m}, 5 \mathrm{H}), 5.88(\mathrm{~s}, 1 \mathrm{H}), 5.24(\mathrm{dd}, J=7.1,4.9 \mathrm{~Hz}$, $1 \mathrm{H}), 4.52(\mathrm{dd}, J=10.9,4.1 \mathrm{~Hz}, 1 \mathrm{H}), 3.72(\mathrm{dd}, J=11.3,4.1 \mathrm{~Hz}, 1 \mathrm{H}), 3.61(\mathrm{dd}, J=11.3,10.9$ $\mathrm{Hz}, 1 \mathrm{H}), 3.52(\mathrm{qd}, J=6.9,4.9 \mathrm{~Hz}, 1 \mathrm{H}), 3.40(\mathrm{~s}, 3 \mathrm{H}), 3.24$ (s, 3H), 2.17 (s, 3H), $1.64-1.46$ (m, 2H), $1.44-1.32(\mathrm{~m}, 1 \mathrm{H}), 1.33(\mathrm{~s}, 3 \mathrm{H}), 1.28(\mathrm{~s}, 3 \mathrm{H}), 1.29-1.18(\mathrm{~m}, 2 \mathrm{H}), 1.16-1.06(\mathrm{~m}$, 2H), $1.04(\mathrm{~d}, J=6.9 \mathrm{~Hz}, 3 \mathrm{H}), 0.99-0.82(\mathrm{~m}, 2 \mathrm{H}), 0.78-0.60(\mathrm{~m}, 2 \mathrm{H}) .{ }^{13} \mathbf{C}$ NMR (101 $\left.\mathrm{MHz}, \mathrm{CDCl}_{3}\right) \delta 209.3,169.9,168.6,134.1,129.1,128.6,127.7,99.8,98.0,78.1,74.3,71.78$, $59.8,48.7,48.1,42.7,39.6,29.2,27.8,26.0,25.8,25.5,20.7,17.8,17.6$, 9.6. FTIR (thin film) $v_{\max } \mathrm{cm}^{-1}$ 2991, 2930, 2854, 1746, 1716, 1453, 1373, 1324, 1231, 1209, 1177, 1141, 1116, 1083, 1057, 1038, 1010, 964, 944, 880, 741, 697. HRMS (ESI-TOF, m/z) calculated for $\mathrm{C}_{28} \mathrm{H}_{40} \mathrm{KO}_{9}{ }^{+}\left([\mathrm{M}+\mathrm{K}]^{+}\right): 559.2304$. Observed: 559.2322. 
$(R)-(2 S, 3 R, E)-1-((2 R, 5 R, 6 R)-5,6-$ dimethoxy-5,6-dimethyl-1,4-dioxan-2-yl)-2-methyl-1oxododec-6-en-3-yl 2-acetoxy-2-phenylacetate $(\boldsymbol{R})-\mathbf{8 h}$.

(8.9 mg from $6.2 \mathrm{mg} \mathbf{7 h}, 98 \%)$.

${ }^{1}$ H NMR $\left(400 \mathrm{MHz}, \mathrm{CDCl}_{3}\right) \delta 7.44-7.36(\mathrm{~m}, 5 \mathrm{H}), 5.83(\mathrm{~s}, 1 \mathrm{H}), 5.46-5.28(\mathrm{~m}, 3 \mathrm{H}), 4.39$ (dd, $J=10.5,4.2 \mathrm{~Hz}, 1 \mathrm{H}), 3.58(\mathrm{dd}, J=11.8,4.2 \mathrm{~Hz}, 1 \mathrm{H}), 3.51(\mathrm{dd}, J=11.8,10.5 \mathrm{~Hz}, 1 \mathrm{H})$, $3.36(\mathrm{~s}, 3 \mathrm{H}), 3.27-3.18(\mathrm{~m}, 4 \mathrm{H}), 2.17$ (s, 3H), $2.09-1.85(\mathrm{~m}, 4 \mathrm{H}), 1.79(\mathrm{~m}, 1 \mathrm{H}), 1.65-1.50$ $(\mathrm{m}, 4 \mathrm{H}), 1.34-1.24(\mathrm{~m}, 3 \mathrm{H}), 1.32(\mathrm{~s}, 3 \mathrm{H}), 1.26(\mathrm{~s}, 3 \mathrm{H}), 0.89$ (t, $J=6.8 \mathrm{~Hz}, 3 \mathrm{H}), 0.77(\mathrm{~d}, J=$ $6.8 \mathrm{~Hz}, 3 \mathrm{H}) .{ }^{13} \mathrm{C}$ NMR $\left(101 \mathrm{MHz}, \mathrm{CDCl}_{3}\right) \delta 208.3,170.3,168.4,133.6,131.9,129.3,128.7$, 128.2, 127.7, 99.7, 98.0, 74.6, 74.0, 71.5, 59.7, 48.8, 48.0, 44.8, 32.7, 32.6, 31.4, 29.2, 28.5, 22.5, 20.7, 17.8, 17.6, 14.0, 8.3. FTIR (thin film) $v_{\max } \mathrm{cm}^{-1} 2990,2950,2925,2872,2855$, 1743, 1716, 1497, 1456, 1373, 1333, 1297, 1261, 1230, 1209, 1175, 1141, 1115, 1083, 1054, 1037, 1004, 968, 944, 879. HRMS (ESI-TOF, m/z) calculated for $\mathrm{C}_{31} \mathrm{H}_{46} \mathrm{KO}_{9}{ }^{+}\left([\mathrm{M}+\mathrm{K}]^{+}\right)$: 601.2773. Observed: 601.2792.

$(S)-(2 S, 3 R, E)-1-((2 R, 5 R, 6 R)-5,6-$ dimethoxy-5,6-dimethyl-1,4-dioxan-2-yl)-2-methyl-1oxododec-6-en-3-yl 2-acetoxy-2-phenylacetate $(\boldsymbol{S})$-8h.

(8.6 mg from $5.9 \mathrm{mg} \mathbf{7 h}, 100 \%)$.

${ }^{1} \mathbf{H}$ NMR $\left(300 \mathrm{MHz}, \mathrm{CDCl}_{3}\right) \delta 7.47-7.35(\mathrm{~m}, 5 \mathrm{H}), 5.83(\mathrm{~s}, 1 \mathrm{H}), 5.39(\mathrm{ddd}, J=8.8,4.5,4.5$ $\mathrm{Hz}, 1 \mathrm{H}), 5.22-5.02(\mathrm{~m}, 2 \mathrm{H}), 4.52(\mathrm{dd}, J=11.0,4.2 \mathrm{~Hz}, 1 \mathrm{H}), 3.73(\mathrm{dd}, J=11.5,4.2 \mathrm{~Hz}, 1 \mathrm{H})$, $3.61(\mathrm{dd}, J=11.5,11.0 \mathrm{~Hz}, 1 \mathrm{H}), 3.35(\mathrm{~s}, 3 \mathrm{H}), 3.34-3.30(\mathrm{~m}, 1 \mathrm{H}), 3.23(\mathrm{~s}, 3 \mathrm{H}), 2.18(\mathrm{~s}, 3 \mathrm{H})$, $1.90-1.81(\mathrm{~m}, 2 \mathrm{H}), 1.66-1.08(\mathrm{~m}, 10 \mathrm{H}), 1.34(\mathrm{~s}, 3 \mathrm{H}), 1.28(\mathrm{~s}, 3 \mathrm{H}), 1.06(\mathrm{~d}, J=6.9 \mathrm{~Hz}$, $3 \mathrm{H}), 0.88(\mathrm{t}, J=6.9 \mathrm{~Hz}, 3 \mathrm{H}) .{ }^{13} \mathrm{C}$ NMR $\left(75 \mathrm{MHz}, \mathrm{CDCl}_{3}\right) \delta 208.7,170.1,168.4,133.8$, $131.8,129.2,128.7,128.0,127.6,99.7,98.0,74.5,73.9,71.9,59.7,48.7,48.1,45.2,32.6$, 32.4, 31.4, 29.1, 28.1, 22.5, 20.7, 17.8, 17.6, 14.1, 9.5. FTIR (thin film) $v_{\max } \mathrm{cm}^{-1} 2989$, 2926, 2872, 2855, 1745, 1716, 1455, 1373, 1333, 1262, 1230, 1209, 1174, 1141, 1116, 1055, 1037, 968, 945, 880. HRMS (ESI-TOF, m/z) calculated for $\mathrm{C}_{31} \mathrm{H}_{46} \mathrm{KO}_{9}{ }^{+}\left([\mathrm{M}+\mathrm{K}]^{+}\right)$: 601.2773. Observed: 601.2797.

(R)-(3R,4S,E)-5-((2R,5R,6R)-5,6-dimethoxy-5,6-dimethyl-1,4-dioxan-2-yl)-4-methyl-5-oxo1-phenylpent-1-en-3-yl 2-acetoxy-2-phenylacetate $(\boldsymbol{R})-\mathbf{8 i}$. 
(8.3 mg from $5.8 \mathrm{mg} \mathbf{7 i}, 97 \%)$.

${ }^{1} \mathbf{H}$ NMR $\left(400 \mathrm{MHz}, \mathrm{CDCl}_{3}\right) \delta 7.48-7.44(\mathrm{~m}, 2 \mathrm{H}), 7.42-7.37(\mathrm{~m}, 3 \mathrm{H}), 7.34-7.23(\mathrm{~m}, 5 \mathrm{H})$, $6.53(\mathrm{dd}, J=16.0,1.2 \mathrm{~Hz}, 1 \mathrm{H}), 6.13(\mathrm{dd}, J=16.0,6.3 \mathrm{~Hz}, 1 \mathrm{H}), 5.92$ (s, 1H), 5.90 (ddd, $J=$ 6.3, 5.9, $1.2 \mathrm{~Hz}, 1 \mathrm{H}), 4.30$ (dd, $J=10.5,4.5 \mathrm{~Hz}, 1 \mathrm{H}), 3.59$ (dd, $J=11.5,4.5 \mathrm{~Hz}, 1 \mathrm{H}), 3.54$ (dd, $J=11.4,10.5 \mathrm{~Hz}, 1 \mathrm{H}), 3.44-3.37$ (m, 1H), 3.29 (s, 3H), 3.20 (s, 3H), 2.18 (s, 3H), 1.35 $(\mathrm{s}, 3 \mathrm{H}), 1.26(\mathrm{~s}, 3 \mathrm{H}), 0.90(\mathrm{~d}, J=6.9 \mathrm{~Hz}, 3 \mathrm{H}) .{ }^{13} \mathbf{C} \mathbf{N M R}\left(101 \mathrm{MHz}, \mathrm{CDCl}_{3}\right) \delta 207.9,170.3$, 168.0, 136.0, 133.6, 133.1, 129.3, 128.8, 128.6, 128.1, 127.6, 126.7, 124.7, 99.8, 98.1, 75.3, 74.6, 72.1, 59.6, 48.7, 48.0, 46.0, 20.6, 17.8, 17.6, 9.9. FTIR (thin film) $v_{\max } \mathrm{cm}^{-1} 2991$, 2943, 2834, 1744, 1716, 1496, 1454, 1373, 1229, 1207, 1169, 1141, 1115, 1054, 1036, 1009, 966, 943, 912, 879. HRMS (ESI-TOF, m/z) calculated for $\mathrm{C}_{30} \mathrm{H}_{40} \mathrm{NO}_{9}{ }^{+}\left(\left[\mathrm{M}+\mathrm{NH}_{4}\right]^{+}\right)$: 558.2698. Observed: 558.2702.

$(S)-(3 R, 4 S, E)-5-((2 R, 5 R, 6 R)-5,6-$ dimethoxy-5,6-dimethyl-1,4-dioxan-2-yl)-4-methyl-5-oxo1-phenylpent-1-en-3-yl 2-acetoxy-2-phenylacetate $(\boldsymbol{S})-\mathbf{8 i}$.

(9.8 mg from $6.1 \mathrm{mg} \mathrm{7i,} \mathrm{98 \% ).}$

${ }^{1} \mathbf{H}$ NMR $\left(400 \mathrm{MHz}, \mathrm{CDCl}_{3}\right) \delta 7.48-7.42(\mathrm{~m}, 2 \mathrm{H}), 7.40-7.36(\mathrm{~m}, 3 \mathrm{H}), 7.28-7.20(\mathrm{~m}, 3 \mathrm{H})$, $7.13(\mathrm{~d}, J=6.7 \mathrm{~Hz}, 2 \mathrm{H}), 6.15(\mathrm{~d}, J=15.1 \mathrm{~Hz}, 1 \mathrm{H}), 5.98-5.88(\mathrm{~m}, 3 \mathrm{H}), 4.44(\mathrm{dd}, J=10.8$, $4.1 \mathrm{~Hz}, 1 \mathrm{H}), 3.72(\mathrm{dd}, J=11.5,4.1 \mathrm{~Hz}, 1 \mathrm{H}), 3.63(\mathrm{dd}, J=11.5,10.8 \mathrm{~Hz}, 1 \mathrm{H}), 3.53-3.45(\mathrm{~m}$, $1 \mathrm{H}), 3.30(\mathrm{~s}, 3 \mathrm{H}), 3.22(\mathrm{~s}, 3 \mathrm{H}), 2.19(\mathrm{~s}, 3 \mathrm{H}), 1.36(\mathrm{~s}, 3 \mathrm{H}), 1.28(\mathrm{~s}, 3 \mathrm{H}), 1.14$ (d, J = $6.9 \mathrm{~Hz}$, 3H). ${ }^{13} \mathrm{C}$ NMR $\left(101 \mathrm{MHz}, \mathrm{CDCl}_{3}\right) \delta 208.3,170.1,167.9,135.9,133.7,132.7,129.3,128.8$, 128.5, 128.0, 127.8, 126.5, 124.5, 99.9, 98.1, 75.2, 74.4, 72.3, 59.7, 48.6, 48.1, 46.0, 20.6, 17.8, 17.6, 10.8. FTIR (thin film) $v_{\max } \mathrm{cm}^{-1} 2990,2943,2834,1747,1717,1496,1455,1373$, 1332, 1230, 1207, 1172, 1142, 1116, 1055, 1036, 1011, 968, 944, 879. HRMS (ESI-TOF, $\mathrm{m} / \mathrm{z})$ calculated for $\mathrm{C}_{30} \mathrm{H}_{36} \mathrm{KO}_{9}{ }^{+}\left([\mathrm{M}+\mathrm{K}]^{+}\right)$: 579.1991. Observed: 579.2011.

(R)-(2S,3R,E)-1-((2R,5R,6R)-5,6-dimethoxy-5,6-dimethyl-1,4-dioxan-2-yl)-2-methyl-1oxooct-4-en-3-yl 2-acetoxy-2-phenylacetate $(\boldsymbol{R})-\mathbf{8 j}$.

(7.5 mg from $5.0 \mathrm{mg} \mathbf{7 j}, 98 \%)$. 
${ }^{1} \mathbf{H}$ NMR $\left(400 \mathrm{MHz}, \mathrm{CDCl}_{3}\right) \delta 7.45-7.35(\mathrm{~m}, 5 \mathrm{H}), 5.87(\mathrm{~s}, 1 \mathrm{H}), 5.72-5.63(\mathrm{~m}, 2 \mathrm{H}), 5.41$ $(\mathrm{dd}, J=15.5,6.7 \mathrm{~Hz}, 1 \mathrm{H}), 4.28(\mathrm{dd}, J=10.5,4.4 \mathrm{~Hz}, 1 \mathrm{H}), 3.59(\mathrm{dd}, J=11.5,4.4 \mathrm{~Hz}, 1 \mathrm{H})$, $3.54(\mathrm{dd}, J=11.5,10.5 \mathrm{~Hz}, 1 \mathrm{H}), 3.32$ (s, 3H), $3.31-3.24(\mathrm{~m}, 1 \mathrm{H}), 3.21(\mathrm{~s}, 3 \mathrm{H}), 2.17$ (s, 3H), $1.98(\mathrm{dt}, J=7.0,7.0, \mathrm{~Hz}, 2 \mathrm{H}), 1.39-1.34(\mathrm{~m}, 2 \mathrm{H}), 1.33(\mathrm{~s}, 3 \mathrm{H}), 1.26(\mathrm{~s}, 3 \mathrm{H}), 0.92-0.80(\mathrm{~m}$, $6 \mathrm{H}) .{ }^{13} \mathrm{C}$ NMR $\left(101 \mathrm{MHz}, \mathrm{CDCl}_{3}\right) \delta 208.1,170.2,167.9,135.5,133.8,129.2,128.7,127.6$, 125.6, 99.8, 98.0, 75.5, 74.6, 71.9, 59.7, 48.7, 48.0, 46.0, 34.2, 22.0, 20.6, 17.8, 17.6, 13.5, 9.8. FTIR (thin film) $v_{\max } \mathrm{cm}^{-1} 2991,2956,2930,2873,2835,1745,1716,1497,1456,1373$, 1336, 1254, 1229, 1208, 1171, 1140, 1115, 1054, 1037, 1010, 967, 943, 879. HRMS (ESITOF, m/z) calculated for $\mathrm{C}_{27} \mathrm{H}_{38} \mathrm{KO}_{9}{ }^{+}\left([\mathrm{M}+\mathrm{K}]^{+}\right): 545.2147$. Observed: 545.2160.

$(S)-(2 S, 3 R, E)-1-((2 R, 5 R, 6 R)-5,6-d i m e t h o x y-5,6-d i m e t h y l-1,4-d i o x a n-2-y l)-2-m e t h y l-1-$ oxooct-4-en-3-yl 2-acetoxy-2-phenylacetate $(\boldsymbol{S}) \mathbf{- 8 j}$.

(7.3 mg from $5.3 \mathrm{mg} \mathbf{7 j}, 90 \%)$.

${ }^{1}$ H NMR $\left(400 \mathrm{MHz}, \mathrm{CDCl}_{3}\right) \delta 7.44-7.33(\mathrm{~m}, 5 \mathrm{H}), 5.87(\mathrm{~s}, 1 \mathrm{H}), 5.67(\mathrm{dd}, J=6.4,6.4 \mathrm{~Hz}$, $1 \mathrm{H}), 5.39$ (ddd, $J=14.8,6.6,6.6 \mathrm{~Hz}, 1 \mathrm{H}), 5.22(\mathrm{dd}, J=14.8,6.4 \mathrm{~Hz}, 1 \mathrm{H}), 4.40(\mathrm{dd}, J=11.1$, $3.9 \mathrm{~Hz}, 1 \mathrm{H}), 3.71(\mathrm{dd}, J=11.1,3.9 \mathrm{~Hz}, 1 \mathrm{H}), 3.63(\mathrm{dd}, J=11.1,11.1 \mathrm{~Hz}, 1 \mathrm{H}), 3.43-3.35(\mathrm{~m}$, $1 \mathrm{H}), 3.33(\mathrm{~s}, 3 \mathrm{H}), 3.23(\mathrm{~s}, 3 \mathrm{H}), 2.17(\mathrm{~s}, 3 \mathrm{H}), 1.88-1.81(\mathrm{dt}, J=7.0,7.0 \mathrm{~Hz}, 2 \mathrm{H}), 1.35(\mathrm{~s}$, $3 \mathrm{H}), 1.28(\mathrm{~s}, 3 \mathrm{H}), 1.22(\mathrm{~m}, 2 \mathrm{H}), 1.08(\mathrm{~d}, J=6.9 \mathrm{~Hz}, 3 \mathrm{H}), 0.76(\mathrm{t}, J=7.3 \mathrm{~Hz}, 3 \mathrm{H}) .{ }^{\mathbf{1 3}} \mathbf{C} \mathbf{~ N M R}$ $\left(101 \mathrm{MHz}, \mathrm{CDCl}_{3}\right) \delta 208.5,170.1,167.9,135.4,133.7,129.2,128.7,127.7,125.2,99.9$, 98.1, 75.6, 74.5, 72.2, 59.7, 48.6, 48.1, 45.8, 34.1, 21.9, 20.6, 17.8, 17.6, 13.4, 10.8. FTIR (thin film) $v_{\max } \mathrm{cm}^{-1}$ 3066, 2956, 1740 (shoulder), 1497, 1456, 1431, 1373, 1328, 1227 (shoulder), 1170, 1141, 1114, 1082, 1048 (shoulder), 1004, 966, 944, 877. HRMS (ESI-TOF, $\mathrm{m} / \mathrm{z}$ ) calculated for $\mathrm{C}_{27} \mathrm{H}_{38} \mathrm{KO}_{9}{ }^{+}\left([\mathrm{M}+\mathrm{K}]^{+}\right)$: 545.2147. Observed: 545.2159.

$(1 S, 2 S)-3-((2 R, 5 R, 6 R)-5,6$-dimethoxy-5,6-dimethyl-1,4-dioxan-2-yl)-2-methyl-3-oxo-1phenylpropyl acetate $\mathbf{1 0 .}$

Acetic anhydride $(111 \mu \mathrm{L}, 1.18 \mathrm{mmol}, 2.0$ equiv) was added dropwise to a stirring solution of the starting material 7a (200 mg, $0.592 \mathrm{mmol}, 1$ equiv) and $N, N$-4-dimethylaminopyridine (361 mg, $2.96 \mathrm{mmol}, 5$ equiv) in dichloromethane $(5 \mathrm{~mL})$ at $0{ }^{\circ} \mathrm{C}$ under an atmosphere of dry argon. The mixture was stirred at this temperature for $15 \mathrm{~min}$. Water $(50 \mathrm{~mL})$ and diethyl 
ether $(10 \mathrm{~mL})$ were added and the mixture was stirred rapidly at room temperature for $2 \mathrm{~min}$. The layers were separated and the aqueous layer was extracted thrice with diethyl ether $(3 \times$ $10 \mathrm{~mL}$ ). The combined organic layers were washed five times with saturated aqueous ammonium chloride $(5 \times 20 \mathrm{~mL})$, water $(10 \mathrm{~mL})$ and brine $(10 \mathrm{~mL})$ and dried over magnesium sulfate. The solution was then filtered through a plug of silica gel ( $20 \mathrm{~g}$, Merck 9385 grade) which was washed several times with diethyl ether. The solvent was removed under reduced pressure to afford the product as a colourless oil (223 mg, 99\%).

${ }^{1}$ H NMR $\left(300 \mathrm{MHz}, \mathrm{CDCl}_{3}\right) \delta 7.36-7.23(\mathrm{~m}, 5 \mathrm{H}), 6.35(\mathrm{~d}, J=5.3 \mathrm{~Hz}, 1 \mathrm{H}), 4.36(\mathrm{dd}, J=$ 10.8, 4.2 Hz, 1H), 3.67-3.62 (m, 2H), 3.55 (dd, $J=11.5,10.8 \mathrm{~Hz}, 1 \mathrm{H}), 3.33$ (s, 3H), 3.23 (s, 3H), 2.09 (s, 3H), 1.42 (s, 3H), 1.28 (s, 3H), 1.03 (d, $J=6.9 \mathrm{~Hz}, 3 \mathrm{H}) .{ }^{13} \mathbf{C}$ NMR $(75 \mathrm{MHz}$, $\left.\mathrm{CDCl}_{3}\right) \delta 209.0,169.9,139.0,128.4,127.9,126.1,99.9,98.0,74.3,72.3,59.5,48.7,48.1$, 47.7, 20.9, 17.8, 17.6, 10.0. FTIR (thin film) $v_{\max } \mathrm{cm}^{-1} 2990,2947,2834,1740,1715,1495$, 1453, 1372, 1228, 1165, 1139, 1113, 1053, 1034, 1022, 969, 944, 878. HRMS (ESI-TOF, $\mathrm{m} / \mathrm{z})$ calculated for $\mathrm{C}_{20} \mathrm{H}_{28} \mathrm{KO}_{7}^{+}\left([\mathrm{M}+\mathrm{K}]^{+}\right)$: 419.1467. Observed: 419.1484.

(1S,2S,4R)-4,5-dihydroxy-2-methyl-3-oxo-1-phenylpentyl acetate 11.

The starting material $10(50.0 \mathrm{mg}, 0.131 \mathrm{mmol})$ was dissolved in tetrahydrofuran $(1 \mathrm{~mL})$ and the solution was stirred at $0{ }^{\circ} \mathrm{C}$ (water/ice bath). To this was added dropwise a mixture of trifluoroacetic acid $(3 \mathrm{~mL})$ and water $(1 \mathrm{~mL})$. The reaction was stirred at $0{ }^{\circ} \mathrm{C}$ for $3 \mathrm{~h}$. The reaction mixture was added portionwise to a stirring mixture of ice (10 g), saturated aqueous sodium bicarbonate $(20 \mathrm{~mL})$ and diethyl ether $(20 \mathrm{~mL})$. The mixture was extracted thrice with diethyl ether $(3 \times 20 \mathrm{~mL})$. The combined organic layers were washed with brine and dried over magnesium sulfate. The solvent was removed under reduced pressure to afford the product as a viscous, colourless oil (33.1 $\mathrm{mg}, 95 \%)$.

${ }^{1}$ H NMR $(400 \mathrm{MHz}, \mathrm{MeOH}) \delta 7.36-7.23(\mathrm{~m}, 5 \mathrm{H}), 6.20(\mathrm{~d}, J=6.8 \mathrm{~Hz}, 1 \mathrm{H}), 4.08(\mathrm{dd}, J=$ $5.3,4.0 \mathrm{~Hz}, 1 \mathrm{H}), 3.65(\mathrm{dq}, J=6.8,6.8 \mathrm{~Hz}, 1 \mathrm{H}), 3.55(\mathrm{dd}, J=11.5,4.0 \mathrm{~Hz}, 1 \mathrm{H}), 3.49(\mathrm{dd}, J=$ 11.5, $5.3 \mathrm{~Hz}, 1 \mathrm{H}), 2.08$ (s, 3H), 1.09 (d, $J=6.8 \mathrm{~Hz}, 3 \mathrm{H}) .{ }^{13} \mathbf{C}$ NMR (101 MHz, MeOH) $\delta$ 214.1, 171.8, 140.6, 129.4, 129.0, 127.7, 78.6, 76.3, 64.5, 48.9, 20.9, 11.7. FTIR (thin film) $v_{\max } \mathrm{cm}^{-1} 3447$ (br), 3033, 2938, 2880, 1715 (has shoulder), 1496, 1455, 1373, 1231 (has 
shoulder), 1117, 1050, 1021, 970, 916, 884, 761. HRMS (ESI-TOF, m/z) calculated for $\mathrm{C}_{14} \mathrm{H}_{18} \mathrm{NaO}_{5}^{+}\left([\mathrm{M}+\mathrm{Na}]^{+}\right): 289.1052$. Observed: 289.1054 .

(2S,3S)-3-acetoxy-2-methyl-3-phenylpropanoic acid $\mathbf{1 2}$.

To a rapidly stirring mixture of the starting keto-diol $11(50 \mathrm{mg}, 0.188 \mathrm{mmol})$ in acetonitrile $(3 \mathrm{~mL})$, dichloromethane $(3 \mathrm{~mL})$ and water $(3 \mathrm{~mL})$ was added sodium periodate $(400 \mathrm{mg}$, $1.87 \mathrm{mmol}, 9.9$ equiv) at $0{ }^{\circ} \mathrm{C}$. The mixture was stirred at this temperature for $20 \mathrm{~min}$. Water $(10 \mathrm{~mL})$ and diethyl ether $(10 \mathrm{~mL})$ were added and the mixture was stirred for $2 \mathrm{~min}$. The layers were separated. The aqueous layer was extracted five times with diethyl ether $(5 \times 5$ $\mathrm{mL})$. The combined organic layers were washed with brine $(10 \mathrm{~mL})$ and dried over magnesium sulfate. The solvent was removed under reduced pressure to afford the product as a paste $(38.8 \mathrm{mg}, 93 \%)$.

${ }^{1}$ H NMR $(400 \mathrm{MHz}, \mathrm{MeOH}) \delta 7.37-7.23(\mathrm{~m}, 5 \mathrm{H}), 6.05(\mathrm{~d}, J=6.7 \mathrm{~Hz}, 1 \mathrm{H}), 2.94(\mathrm{dq}, J=$ $6.9 \mathrm{~Hz}, 1 \mathrm{H}), 2.08(\mathrm{~s}, 3 \mathrm{H}), 1.19$ (d, $J=7.0 \mathrm{~Hz}, 3 \mathrm{H}) .{ }^{13} \mathrm{C}$ NMR $(101 \mathrm{MHz}, \mathrm{MeOH}) \delta$ 176.7, 171.7, 140.2, 129.4, 129.0, 127.7, 77.5, 46.8, 20.8, 12.8. FTIR (thin film) $v_{\max } \mathrm{cm}^{-1} 3032$, 2983, 2945, 1736, 1710, 1496, 1431, 1456, 1373, 1227, 1116, 1095, 1021, 973, 917, 847. HRMS (ESI-TOF, m/z) calculated for $\mathrm{C}_{12} \mathrm{H}_{14} \mathrm{NaO}_{4}{ }^{+}\left([\mathrm{M}+\mathrm{Na}]^{+}\right)$: 245.0790. Observed: 245.0795 .

(2S,3S)-3-hydroxy-2-methyl-3-phenylpropanoic acid 13.

To a solution of the starting acetate $12(26.0 \mathrm{mg}, 0.117 \mathrm{mmol}, 1$ equiv) in tetrahydrofuran (18 $\mathrm{mL}$ ) was added an aqueous solution of lithium hydroxide $(0.1 \mathrm{M}, 4.5 \mathrm{~mL}, 0.450 \mathrm{mmol}, 3.85$ equiv) at room temperature. The mixture was stirred at room temperature overnight. Ether $(20 \mathrm{~mL})$ and dilute aqueous hydrochloric acid $(1 \mathrm{M}, 20 \mathrm{~mL})$ were added and the mixture was stirred for 1 minute before separating the layers. The aqueous layer was extracted thrice with diethyl ether $(3 \times 20 \mathrm{~mL})$. The combined organic layers were dried over magnesium sulfate. The solvent was removed under reduced pressure to afford the product as a white paste (18.6 $\mathrm{mg}, 88 \%)$. 
${ }^{1} \mathbf{H}$ NMR $\left(300 \mathrm{MHz}, \mathrm{CDCl}_{3}\right) \delta 7.38-7.27(\mathrm{~m}, 5 \mathrm{H}), 5.19(\mathrm{~d}, J=3.9 \mathrm{~Hz}, 1 \mathrm{H}), 2.84(\mathrm{qd}, J=$ 7.2, $3.9 \mathrm{~Hz}, 1 \mathrm{H}), 1.15(\mathrm{~d}, J=7.2 \mathrm{~Hz}, 3 \mathrm{H}) .{ }^{13} \mathbf{C} \mathbf{N M R}\left(75 \mathrm{MHz}, \mathrm{CDCl}_{3}\right) \delta 180.6,141.0,128.4$, 127.7, 125.9, 73.3, 46.1, 10.3. FTIR (thin film) $v_{\max } \mathrm{cm}^{-1} 3600-3000$ (v. br), 3063, 3031, 2983, 2924, 1704, 1494, 1454, 1402, 1196, 1122, 1060, 1021, 984, 909. HRMS (ESI-TOF, $\mathrm{m} / \mathrm{z}$ ) calculated for $\mathrm{C}_{10} \mathrm{H}_{12} \mathrm{NaO}_{3}{ }^{+}\left([\mathrm{M}+\mathrm{Na}]^{+}\right):$203.0679. Observed: 203.0676. $[\alpha]_{\mathrm{d}}=-22.0(\mathrm{c}$ $\left.=0.9, \mathrm{CH}_{2} \mathrm{Cl}_{2}, \alpha=-0.20,1=10 \mathrm{~cm}\right)$.

\section{References:}

1 Borodin, A. Journal für Praktische Chemie 1864, 93, 413.

2 Wurtz, A. Journal für Praktische Chemie 1872, 5, 457.

3 (a) Mahrwald, R. E. Modern Aldol Reactions; Wiley-VCH, Weinheim, 2004; (b) Kobayashi, S.; Fujishita, Y.; Mukaiyama, T. Chem. Lett. 1990, 19, 1455; (c) Mukaiyama, T. Org. React. 1982, 28, 203; (d) Mukaiyama, T.; Banno, K.; Narasaka, K. J. Amer. Chem. Soc. 1974, 96, 7503; (e) Johnson, J. S.; Evans, D. A. Acc. Chem. Res. 2000, 33, 325; (f) Evans, D. A.; Ng, H. P.; Clark, J. S.; Rieger, D. L. Tetrahedron 1992, 48, 2127; (g) Evans, D. A.; Rieger, D. L.; Bilodeau, M. T.; Urpi, F. J. Amer. Chem. Soc. 1991, 113, 1047; (h) Evans, D. A.; Bartroli, J.; Shih, T. L. J. Amer. Chem. Soc. 1981, 103, 2127; (i) Reetz, M. T.; Kunisch, F.; Heitmann, P. Tetrahedron Lett. 1986, 27, 4721; (j) Crimmins, M. T.; King, B. W.; Tabet, E. A.; Chaudhary, K. J. Org. Chem. 2001, 66, 894; (k) Van Draanen, N. A.; Arseniyadis, S.; Crimmins, M. T.; Heathcock, C. H. J. Org. Chem. 1991, 56, 2499; (l) Oppolzer, W.; Blagg, J.; Rodriguez, I.; Walther, E. J. Amer. Chem. Soc. 1990, 112, 2767; (m) Notz, W.; Tanaka, F.; Barbas, C. F. Acc. Chem. Res. 2004, 37, 580; (n) Carreira, E. M.; Singer, R. A.; Lee, W. J. Amer. Chem. Soc. 1994, 116, 8837; (o Denmark, S. E.; Stavenger, R. A. Acc. Chem. Res. 2000, 33, 432; (p) Yoshikawa, N.; Yamada, Y. M. A.; Das, J.; Sasai, H.; Shibasaki, M. J. Amer. Chem. Soc. 1999, 121, 4168; (q) Notz, W.; List, B. J. Amer. Chem. Soc. 2000, 122, 7386; (r) Northrup, A. B.; MacMillan, D. W. C. J. Amer. Chem. Soc. 2002, 124, 6798; (s) Dias, L. C.; Aguilar, A. M. Chem. Soc. Rev. 2008, 37, 451; (t) Palomo, C.; Oiarbide, M.; Garcia, J. M. Chem. Soc. Rev. 2004, 33, 65; (u) Matsuo, J. i.; Murakami, M. Angew. Chem. Int. Ed. 2013, 52, 9109. 
(a) Kan, S. B. J.; Ng, K. K. H.; Paterson, I. Angew. Chem. Int. Ed. 2013, 52, 9097; (b) Schetter, B.; Mahrwald, R. Angew. Chem. Int. Ed. 2006, 45, 7506; (c) Evans, D. A.; Kaldor, S. W.; Jones, T. K.; Clardy, J.; Stout, T. J. J. Amer. Chem. Soc. 1990, 112, 7001; (d) Evans, D. A.; Fitch, D. M.; Smith, T. E.; Cee, V. J. J. Amer. Chem. Soc. 2000, 122, 10033; (e) Crimmins, M. T.; Slade, D. J. Org. Lett. 2006, 8, 2191; (f) Paterson, I.; Delgado, O.; Florence, G. J.; Lyothier, I.; O'Brien, M.; Scott, J. P.; Sereinig, N. J. Org. Chem. 2005, 70, 150; (g) Paterson, I.; Anderson, E. A.; Dalby, S. M.; Lim, J. H.; Genovino, J.; Maltas, P.; Moessner, C. Angew. Chem. Int. Ed. 2008, 47, 3016; (h) Trost, B. M.; O’Boyle, B. M. J. Amer. Chem. Soc. 2008, 130, 16190; (i) Packard, G. K.; Hu, Y.; Vescovi, A.; Rychnovsky, S. D. Angew. Chem. Int. Ed. 2004, 43, 2822; (j) Kraus, H.; Francais, A.; O'Brien, M.; Frost, J.; Dieguez-Vazquez, A.; Polara, A.; Baricordi, N.; Horan, R.; Hsu, D.-S.; Tsunoda, T.; Ley, S. V. Chem. Sci. 2013, 4, 1989; (k) Paterson, I.; Chen, D. Y. K.; Coster, M. J.; Aceña, J. L.; Bach, J.; Gibson, K. R.; Keown, L. E.; Oballa, R. M.; Trieselmann, T.; Wallace, D. J.; Hodgson, A. P.; Norcross, R. D. Angew. Chem. Int. Ed. 2001, 40, 4055; (1) Gaunt, M. J.; Jessiman, A. S.; Orsini, P.; Tanner, H. R.; Hook, D. F.; Ley, S. V. Org. Lett. 2003, 5,4819 .

5 (a) Ley, S. V.; Polara, A. J. Org. Chem. 2007, 72, 5943; (b) Tzschucke, C. C.; Pradidphol, N.; Diéguez-Vázquez, A.; Kongkathip, B.; Kongkathip, N.; Ley, S. V. SynLett 2008, 2008, 1293; (c) Dixon, D. J.; Ley, S. V.; Lohmann, S.; Sheppard, T. D. SynLett 2005, 2005, 481; (d) Ley, S. V.; Dixon, D. J.; Guy, R. T.; Rodriguez, F.; Sheppard, T. D. Org. Biomol. Chem. 2005, 3, 4095; (e) Ley, S. V.; Diez, E.; Dixon, D. J.; Guy, R. T.; Michel, P.; Nattrass, G. L.; Sheppard, T. D. Org. Biomol. Chem. 2004, 2, 3608; (f) Ley, S. V.; Dixon, D. J.; Guy, R. T.; Palomero, M. A.; Polara, A.; Rodriguez, F.; Sheppard, T. D. Org. Biomol. Chem. 2004, 2, 3618; (g) Dixon, D. J.; Guarna, A.; Ley, S. V.; Polara, A.; Rodríguez, F. Synthesis 2002, 2002, 1973; (h) Dixon, D. J.; Ley, S. V.; Polara, A.; Sheppard, T. Org. Lett. 2001, 3, 3749; (i) Ley, S. V.; Tackett, M. N.; Maddess, M. L.; Anderson, J. C.; Brennan, P. E.; Cappi, M. W.; Heer, J. P.; Helgen, C.; Kori, M.; Kouklovsky, C.; Marsden, S. P.; Norman, J.; Osborn, D. P.; Palomero, M. Á.; Pavey, J. B. J.; Pinel, C.; Robinson, L. A.; Schnaubelt, J.; Scott, J. S.; Spilling, C. D.; Watanabe, H.; Wesson, K. E.; Willis, M. C. Chem.-Eur. J. 2009, 15, 2874. 
(a) Ley, S. V.; Michel, P. Synthesis 2004, 2004, 147; (b) Michel, P.; Ley, S. V. Angew. Chem. Int. Ed. 2002, 41, 3898.

7 Knudsen, K. R.; Stepan, A. F.; Michel, P.; Ley, S. V. Org. Biomol. Chem. 2006, 4, 1471.

8 Cowden, C. J.; Paterson, I. Org. React. 1997, 51, 1.

9 (a) Stiles, M.; Winkler, R. R.; Chang, Y.-L.; Traynor, L. J. Amer. Chem. Soc. 1964, 86, 3337; (b) House, H. O.; Crumrine, D. S.; Teranishi, A. Y.; Olmstead, H. D. J. Amer. Chem. Soc. 1973, 95, 3310; (c) Heathcock, C. H.; Buse, C. T.; Kleschick, W. A.; Pirrung, M. C.; Sohn, J. E.; Lampe, J. J. Org. Chem. 1980, 45, 1066.

10 Paterson, I.; Wallace, D. J.; Velázquez, S. M. Tetrahedron Lett. 1994, 35, 9083.

11 Masamune, S.; Choy, W.; Kerdesky Francis, A. J.; Imperiali, B. J. Amer. Chem. Soc. 1981, 103, 1566.

12 (a) Gage, J. R.; Evans, D. A. Org. Synth. 1990, 68, 77; (b) Maeng, Y. H.; Jun, J. G. Bull. Kor. Chem. Soc. 2004, 25, 143. 